IEKP-KA/96-07

KA-TP-18-96

hep-ph/9607286

July 7 th, 1996

\title{
Global Fits of the SM and MSSM to Electroweak Precision Data
}

\author{
W. de Boer ${ }^{1}$, A. Dabelstein ${ }^{3 \dagger}$, W. Hollik ${ }^{2 \ddagger}$ \\ W. Mösle ${ }^{2 \S}$ U. Schwickerath ${ }^{19}$, \\ 1) Inst. für Experimentelle Kernphysik, Univ. of Karlsruhe, \\ Postfach 6980, D-76128 Karlsruhe, Germany \\ 2) Inst. für Theoretische Physik, Univ. of Karlsruhe, \\ Postfach 6980, D-76128 Karlsruhe, Germany \\ 3) Inst. für Theoretische Physik, Univ. of München, \\ James Franck Straße, D-85747 Garching, Germany
}

\begin{abstract}
The Minimal supersymmetric extension of the Standard Model (MSSM) with light stops, charginos or pseudoscalar Higgs bosons has been suggested as an explanation of the too high value of the branching ratio of the $Z^{0}$ boson into $b$ quarks ( $R_{b}$ anomaly). A program including all radiative corrections to the MSSM at the same level as the radiative corrections to the SM has been developed and used to perform global fits to all electroweak data from LEP, SLC and the Tevatron. The probability of the global fit improves from $8 \%$ in the SM to $18 \%$ in the MSSM. Including the $b \rightarrow s \gamma$ rate, as measured by CLEO, reduces the probability from $18 \%$ to $15 \%$. In the constrained MSSM requiring unification and electroweak symmetry breaking no improvement of $R_{b}$ is possible.
\end{abstract}

\footnotetext{
*Email: wim.de.boer@cern.ch

${ }^{\dagger}$ E-mail: Andreas.Dabelstein@tu-muenchen.de

${ }^{\ddagger}$ E-mail: hollik@itpaxp3.physik.uni-karlsruhe.de

§E-mail: wm@itpaxp1.physik.uni-karlsruhe.de

IE-mail: Ulrich.Schwickerath@cern.ch
} 


\section{Introduction}

Present LEP data show a too high value of $R_{b}(3.4 \sigma)$ and a too low value of $R_{c}$ $(2 \sigma)$ where $R_{b(c)}$ is the ratio $R_{b(c)}=\Gamma_{Z^{0} \rightarrow b \bar{b}(c \bar{c})} / \Gamma_{Z^{0} \rightarrow q \bar{q}}$. In the past it has been shown by several groups that it is possible to improve $R_{b}$ using supersymmetric models with light charginos, stops or Higgses, which yield positive contributions to the $Z b \bar{b}$ vertex [1]-9]. In this paper we perform an equivalent analysis of all electroweak data both in the Standard Model (SM) and its supersymmetric extension (MSSM). The conclusion is that only a moderate increase in the probability can be found for the MSSM as compared to the SM, if all present limits on supersymmetric particles and the ratio $b \rightarrow s \gamma$ are taken into account. The analysis was performed using all actual electroweak data from Tevatron [11], 12], LEP and SLC [13, 14], the measurement

of $\frac{B R(b \rightarrow s \gamma)}{B R(b \rightarrow c e \bar{\nu})}$ from CLEO 15] and limits on the masses of supersymmetric particles [1, 16, 17, 18, 19, 20].

For low values of $\tan \beta$ the diagrams with charginos and right handed stops in the vertex loop are dominant, while for high $\tan \beta$ the exchange of the pseudoscalar Higgs between the outgoing $b$-quarks, which is proportional to $m_{b} \cdot \tan \beta$ becomes important too. It should be noted that light stops and charginos will contribute to the $b \rightarrow s \gamma$ rate with an opposite sign as the SM diagram of the top - W boson loop, so the predicted $b \rightarrow s \gamma$ rate can become easily too small, if the stops and charginos are light. Therefore, getting $R_{b}$ and $b \rightarrow s \gamma$ right is not easy, since $R_{b}$ wants sparticle masses near the experimental limits, while $b \rightarrow s \gamma$ needs sparticles well above the experimental limits or a sufficiently large $\tan \beta$.

\section{2. $Z^{0}$ boson on-resonance observables}

At the $Z$ boson resonance two classes of precision observables are available:

a) inclusive quantities:

- the partial leptonic and hadronic decay width $\Gamma_{f \bar{f}}$,

- the total decay width $\Gamma_{Z}$,

- the hadronic peak cross section $\sigma_{h}$,

- the ratio of the hadronic to the electronic decay width of the $Z$ boson: $R_{h}$,

- the ratio of the partial decay width for $Z \rightarrow c \bar{c}(b \bar{b})$ to the hadronic width, $R_{c}, R_{b}$.

b) asymmetries and the corresponding mixing angles:

- the forward-backward asymmetries $A_{F B}^{f}$,

- the left-right asymmetries $A_{L R}^{f}$, 
- the $\tau$ polarization $P_{\tau}$,

- the effective weak mixing angles $\sin ^{2} \theta_{\text {eff }}^{f}$.

Together with the quantity $\Delta r$ in the correlation of the $W$ mass to the electroweak input parameters $G_{\mu}, M_{Z}$ and $\alpha_{e m}$, this set of precision observables is convenient for a numerical analysis of the supersymmetric parameter space. In the following the observables defined above are expressed with the help of effective couplings.

\subsection{The effective $Z$ - $f$ - $f$ couplings}

The coupling of the $Z$ boson to fermions $f$ can be expressed by effective vector and axial vector coupling constants $v_{e f f}^{f}, a_{\text {eff }}^{f}$ in terms of the NC vertex:

$$
J_{N C}^{\mu}=\frac{e}{2 s_{W}^{2} c_{W}^{2}} \gamma^{\mu}\left(v_{e f f}^{f}-a_{e f f}^{f} \gamma_{5}\right)
$$

where the convention is introduced : $c_{W}^{2}=\cos ^{2} \theta_{W}=1-s_{W}^{2}=M_{W}^{2} / M_{Z}^{2}$ [21]. Input parameters are the $\mu$ decay constant $G_{\mu}=1.166392 \times 10^{-5} \mathrm{GeV}^{-2}, \alpha_{E M}=1 / 137.036$ and the mass of the $Z^{0}$ boson $M_{Z}=91.1884 \mathrm{GeV}$. The mass of the $W$ boson is related to these input parameters through:

$$
\frac{G_{\mu}}{\sqrt{2}}=\frac{\pi \alpha_{E M}}{2 s_{W}^{2} M_{W}^{2}} \cdot \frac{1}{1-\Delta r_{M S S M}\left(\alpha_{E M}, M_{W}, M_{Z}, m_{t}, \ldots\right)},
$$

where the complete MSSM one-loop contributions are parameterized by the quantity $\Delta r_{M S S M}$ [22]. Leading higher order Standard Model corrections [23, 24] to the quantity $\Delta r$ are included in the calculation.

The effective couplings $v_{e f f}^{f}, a_{e f f}^{f}$ can be written as:

$$
\begin{aligned}
& v_{e f f}^{f}=\sqrt{Z_{Z}}\left(v^{f}+\Delta v^{f}+Z_{M} Q_{f}\right) \\
& a_{e f f}^{f}=\sqrt{Z_{Z}}\left(a^{f}+\Delta a^{f}\right) .
\end{aligned}
$$

$v^{f}$ and $a^{f}$ are the tree-level vector and axial vector couplings:

$$
v^{f}=I_{3}^{f}-2 Q_{f} s_{W}^{2}, a^{f}=I_{3}^{f} .
$$

$Z_{Z}, Z_{M}$ are given in eq. (10). The complete MSSM one-loop contributions of the nonuniversal finite vector and axial vector couplings $\Delta v^{f}, \Delta a^{f}$ have been calculated [25], together with the leading two-loop Standard Model contributions [23, 24, 26]. They are derived in the 't Hooft-Feynman gauge and in the on-shell renormalization scheme 27. Fig. 1 shows the MSSM one-loop $Z \rightarrow f \bar{f}$ vertex correction diagrams. 

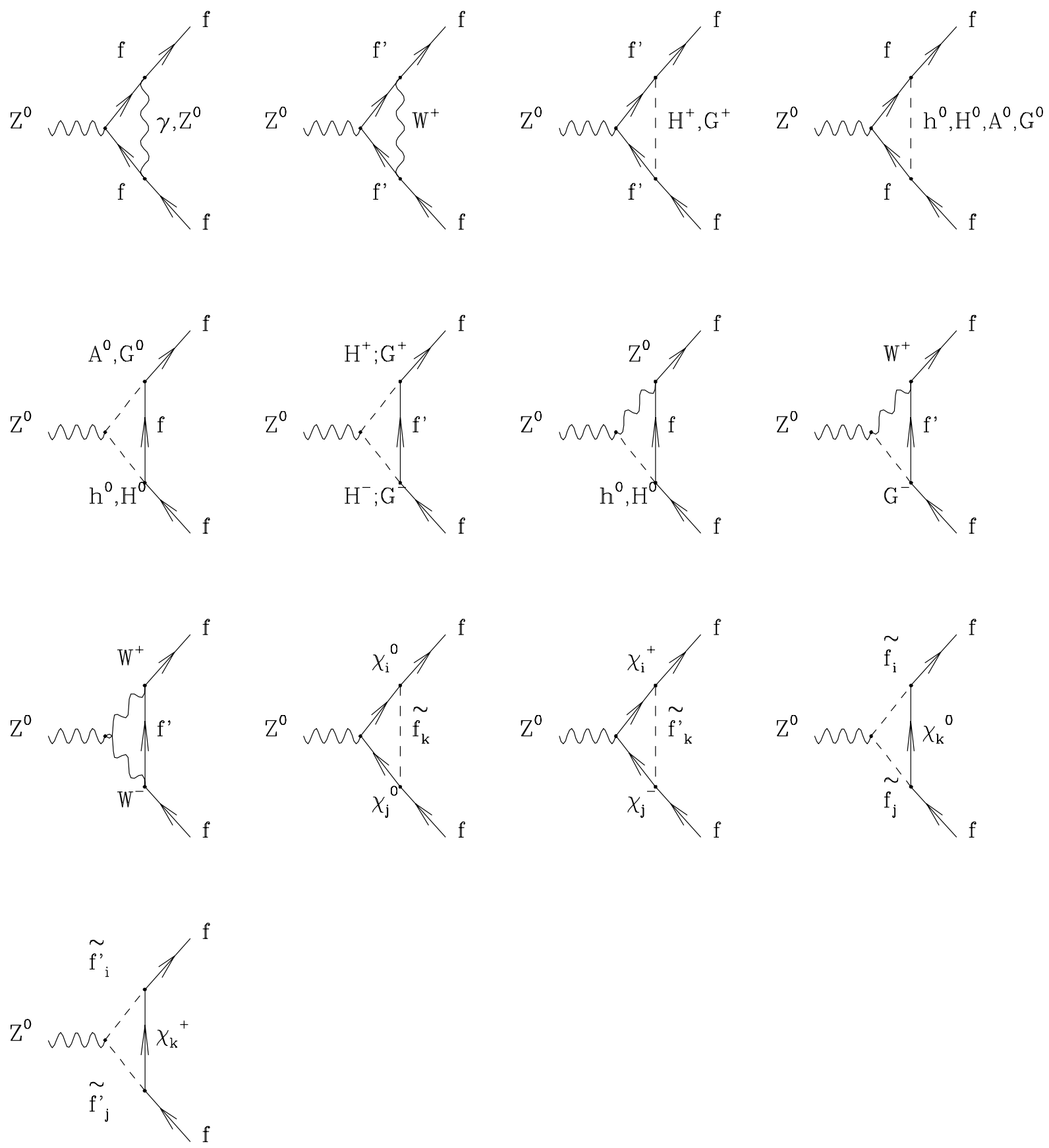

Figure 1: MSSM one-loop $Z \rightarrow f \bar{f}$ vertex correction diagrams. $i, j, k=1, . ., 2(4)$ are chargino, neutralino and sfermions indices. No particle permutations are shown. 

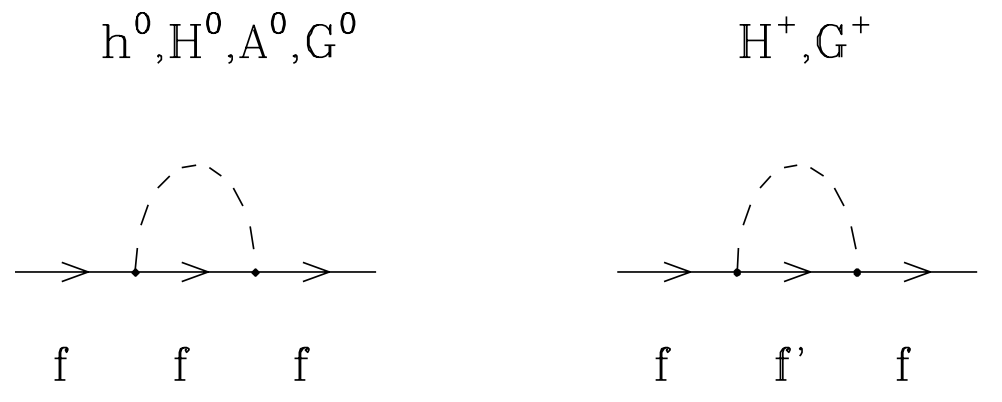

Z, $\gamma$
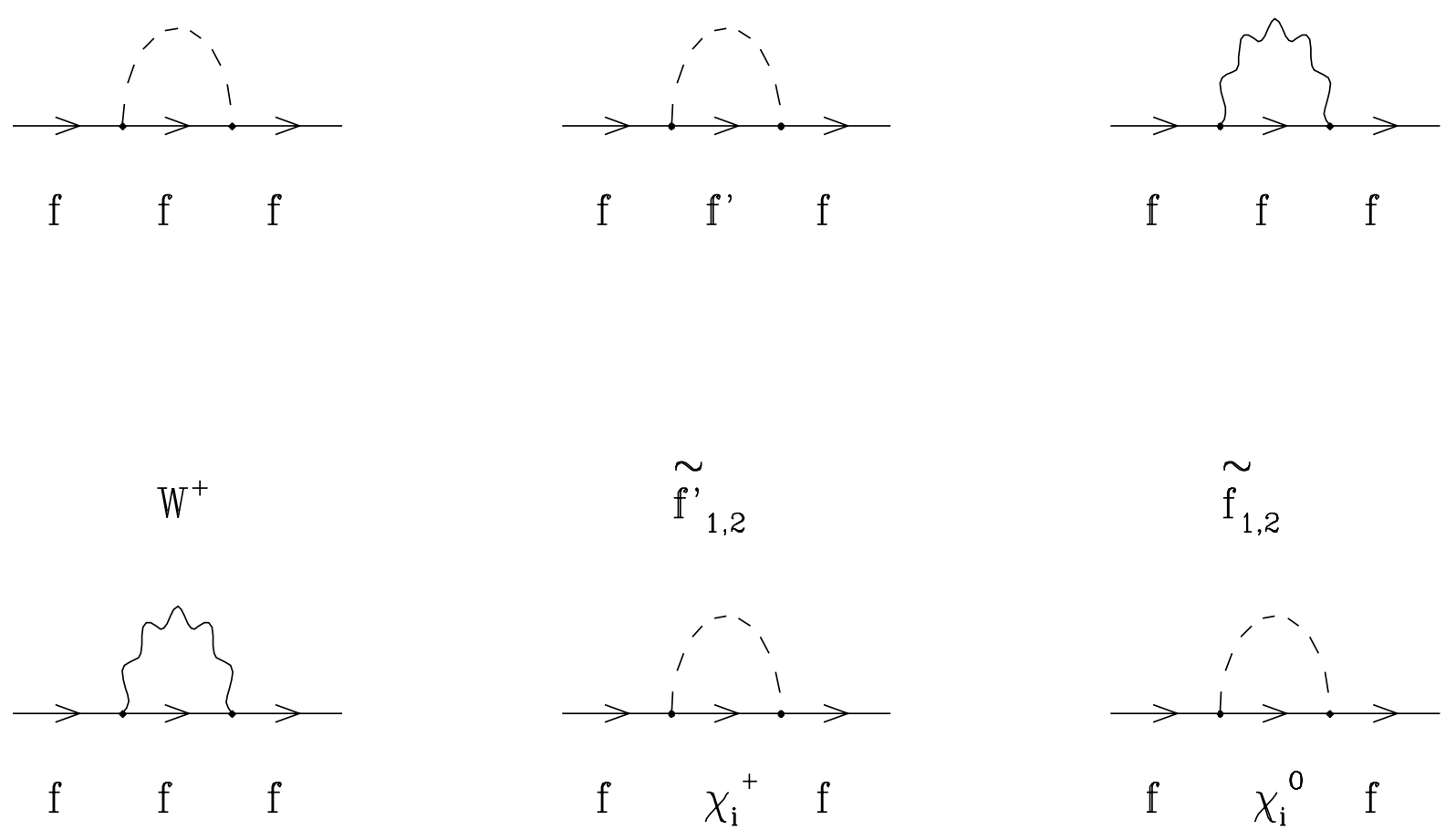

Figure 2: MSSM fermion self-energies.
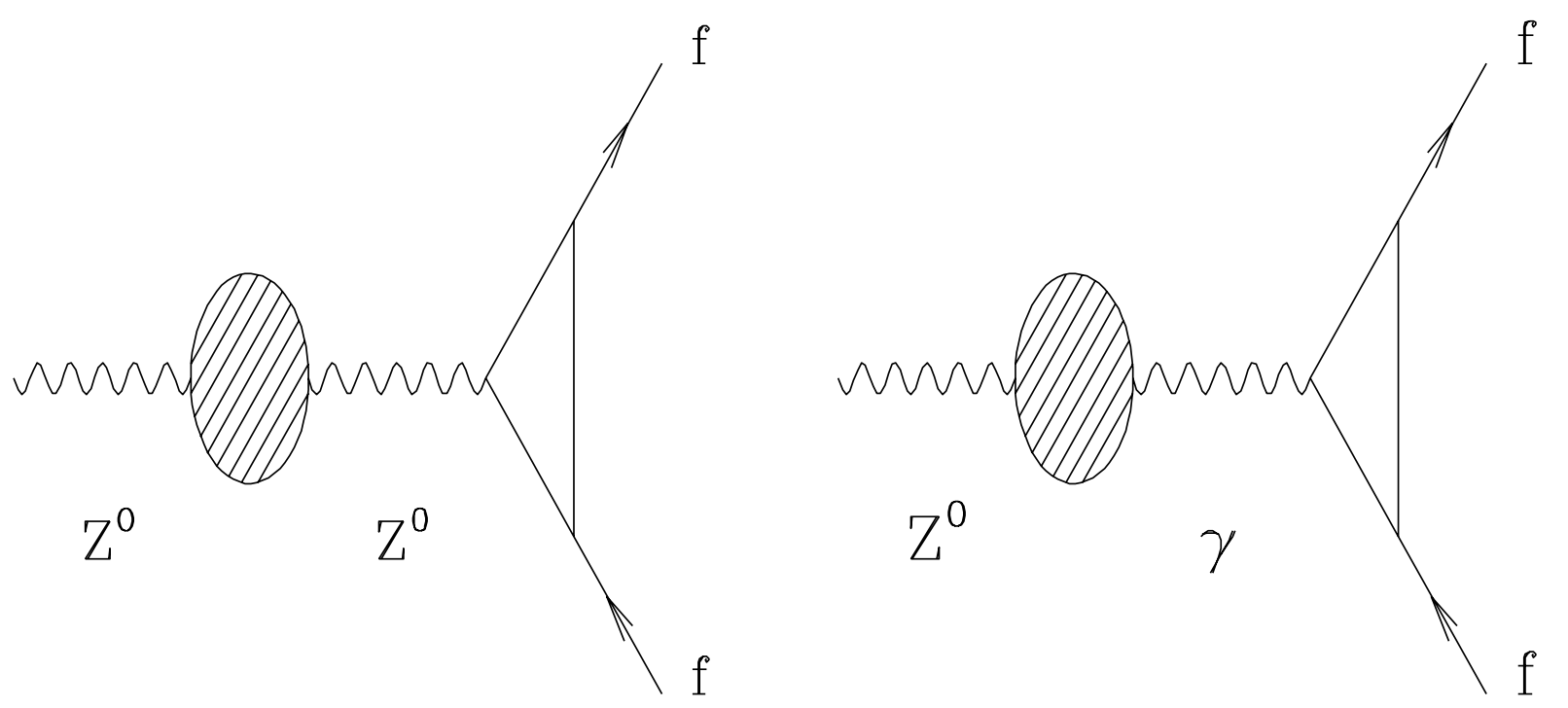

Figure 3: $Z$ boson wave function renormalization. 
The non-universal contributions can be written in the following way:

$$
\begin{aligned}
& \Delta v_{f}=F_{V}^{S M}+\Delta F_{V}, \\
& \Delta a_{f}=F_{A}^{S M}+\Delta F_{A} .
\end{aligned}
$$

The Standard Model form factors $F_{V, A}^{S M}$ corresponding to the diagrams of figs. 1 and 2 can be found e.g in refs. 227, 23]. The diagrams with a virtual photon are listed for completeness in the figures. They are not part of the effective weak couplings but are treated separately in the QED corrections, together with real photon bremsstrahlung. The non-standard contributions are summarized by

$$
\begin{aligned}
\Delta F_{V} & =\sum_{i} F_{V}^{(i)}+v_{f} \delta Z_{V}^{f}+a_{f} \delta Z_{A}^{f} \\
\Delta F_{A} & =\sum_{i} F_{A}^{(i)}+v_{f} \delta Z_{A}^{f}+a_{f} \delta Z_{V}^{f}
\end{aligned}
$$

where the sum extends over the diagrams of fig. 1 with internal charged and neutral Higgs bosons, charginos, neutralinos and scalar fermions, each diagram contributing

$$
F_{V}^{(i)} \gamma_{\mu}-F_{A}^{(i)} \gamma_{\mu} \gamma_{5}
$$

to the $Z f f$-vertex. The self-energy diagrams of fig. 2 with internal neutral Higgs, chargino, neutralino and sfermion lines determine the field renormalization constants

$$
\begin{aligned}
\delta Z_{V}^{f} & =-\Sigma_{V}\left(m_{f}^{2}\right)-2 m_{f}^{2}\left[\Sigma_{V}^{\prime}\left(m_{f}^{2}\right)+\Sigma_{s}^{\prime}\left(m_{f}^{2}\right)\right] \\
\delta Z_{A}^{f} & =\Sigma\left(m_{f}^{2}\right)
\end{aligned}
$$

with the scalar functions $\Sigma_{V, A, S}$ in the decomposition of the fermion self-energy according to

$$
\Sigma=\not p \Sigma_{V}+\not p \gamma_{5} \Sigma_{A}+m_{f} \Sigma_{S}
$$

The contributions from the Higgs sector are given explicitly in ref. [28]. For the genuine SUSY diagrams, the couplings for charginos, neutralinos and sfermions are taken from [30], together with the diagonalization matrices given in section 3 .

The universal propagator corrections from the finite $Z$ boson wave function renormalization $Z_{Z}$ and the $\gamma Z$ mixing $Z_{M}$ are derived from the $(\gamma, Z)$ propagator matrix. The inverse matrix is:

$$
\left(\Delta_{\mu \nu}\right)^{-1}=i g_{\mu \nu}\left(\begin{array}{ll}
k^{2}+\hat{\Sigma}_{\gamma}\left(k^{2}\right) & \hat{\Sigma}_{\gamma Z}\left(k^{2}\right) \\
\hat{\Sigma}_{\gamma Z}\left(k^{2}\right) & k^{2}-M_{Z}^{2}+\hat{\Sigma}_{Z}\left(k^{2}\right)
\end{array}\right),
$$

where $\hat{\Sigma}_{\gamma}, \hat{\Sigma}_{Z}, \hat{\Sigma}_{\gamma Z}$ are the renormalized self energies and mixing. They are obtained by summing the loop diagrams, shown symbolically in fig. 3 , and the counter terms and can be found in ref. [29. 
The entries in the $(\gamma, Z)$ propagator matrix:

$$
\Delta_{\mu \nu}=-i g_{\mu \nu}\left(\begin{array}{cc}
\Delta_{\gamma} & \Delta_{\gamma Z} \\
\Delta_{\gamma Z} & \Delta_{Z}
\end{array}\right)
$$

are given by:

$$
\begin{aligned}
\Delta_{\gamma}\left(k^{2}\right) & =\frac{1}{k^{2}+\hat{\Sigma}_{\gamma}\left(k^{2}\right)-\frac{\hat{\Sigma}_{\gamma Z}^{2}\left(k^{2}\right)}{k^{2}-M_{Z}^{2}+\hat{\Sigma}_{Z}\left(k^{2}\right)}} \\
\Delta_{Z}\left(k^{2}\right) & =\frac{1}{k^{2}-M_{Z}^{2}+\hat{\Sigma}_{Z}\left(k^{2}\right)-\frac{\hat{\Sigma}_{\gamma Z}^{2}\left(k^{2}\right)}{k^{2}+\hat{\Sigma}_{\gamma}\left(k^{2}\right)}} \\
\Delta_{\gamma Z}\left(k^{2}\right) & =-\frac{\hat{\Sigma}_{\gamma Z}\left(k^{2}\right)}{\left[k^{2}+\hat{\Sigma}_{\gamma}\left(k^{2}\right)\right]\left[k^{2}-M_{Z}^{2}+\hat{\Sigma}_{Z}\left(k^{2}\right)\right]-\hat{\Sigma}_{\gamma Z}^{2}\left(k^{2}\right)} .
\end{aligned}
$$

The renormalization condition to define the mass of the $Z$ boson is given by the pole of the propagator matrix (eq. 6). The pole $k^{2}=M_{Z}^{2}$ is the solution of the equation:

$$
\mathcal{R e}\left[\left(\mathrm{M}_{\mathrm{Z}}^{2}+\hat{\Sigma}_{\gamma}\left(\mathrm{M}_{\mathrm{Z}}^{2}\right)\right) \hat{\Sigma}_{\mathrm{Z}}\left(\mathrm{k}^{2}\right)-\hat{\Sigma}_{\gamma \mathrm{Z}}^{2}\left(\mathrm{M}_{\mathrm{Z}}^{2}\right)\right]=0 .
$$

Eq. (8) yields the wave function renormalization $Z_{Z}$ and mixing $Z_{M}$ :

$$
\begin{aligned}
Z_{Z} & =\operatorname{Res}_{M_{Z}} \Delta_{Z}=\left.\frac{1}{1+\mathcal{R e} \hat{\Sigma}_{Z}^{\prime}\left(\mathrm{k}^{2}\right)-\mathcal{R e}\left(\frac{\hat{\Sigma}_{\gamma Z}^{2}\left(\mathrm{k}^{2}\right)}{\mathrm{k}^{2}+\hat{\Sigma}_{\gamma}\left(\mathrm{k}^{2}\right)}\right)^{\prime}}\right|_{k^{2}=M_{Z}^{2}} \\
Z_{M} & =-\frac{\hat{\Sigma}_{\gamma Z}\left(M_{Z}^{2}\right)}{M_{Z}^{2}+\hat{\Sigma}_{\gamma}\left(M_{Z}^{2}\right)} .
\end{aligned}
$$

\section{2. $Z$ boson observables}

The fermionic $Z$ boson partial decay widths $\Gamma_{f \bar{f}}$ can be written as follows:

1) $f \neq b$ :

$$
\begin{aligned}
\Gamma_{f \bar{f}}= & \frac{N_{C} \sqrt{2} G_{\mu} M_{Z}^{3}}{12 \pi}\left(1-\Delta r_{M S S M}\right)\left[\left(v_{e f f}^{f}\right)^{2}+\left(a_{e f f}^{f}\right)^{2}\left(1-\frac{6 m_{f}^{2}}{M_{Z}^{2}}\right)\right] . \\
& \cdot\left(1+\frac{3 \alpha_{E M}}{4 \pi} Q_{f}^{2}\right)\left(1+\delta_{Q C D}^{f}\right)
\end{aligned}
$$

where

$$
\delta_{Q C D}^{f}=\left\{\begin{array}{ll}
0 & , f=\text { leptons } \\
\frac{\alpha_{s}}{\pi}+1.405\left(\frac{\alpha_{s}}{\pi}\right)^{2}-12.8\left(\frac{\alpha_{s}}{\pi}\right)^{3}-\frac{Q_{f}^{2}}{4} \frac{\alpha \alpha_{s}}{\pi^{2}} & , f=\text { quarks }
\end{array} .\right.
$$


2) $f=b$ :

$$
\begin{aligned}
\Gamma_{b \bar{b}}= & \frac{N_{C} \sqrt{2} G_{\mu} M_{Z}^{3}}{12 \pi}\left(1-\Delta r_{M S S M}\right)\left[\left(v_{e f f}^{b}\right)^{2}+\left(a_{e f f}^{b}\right)^{2}\right] \\
& \cdot\left(1+\frac{3 \alpha_{E M}}{4 \pi} Q_{b}^{2}\right)\left(1+\delta_{Q C D}^{b}\right)+\Delta \Gamma_{b \bar{b}} .
\end{aligned}
$$

In $\Delta \Gamma_{b \bar{b}}$ the $b$ quark specific finite mass terms with QCD corrections [26] are included. $\delta_{Q C D}^{b}$ is given in eq. (12).

The total decay width $\Gamma_{Z}$ is the sum of the contributions from leptons and quarks:

$$
\Gamma_{Z}=\sum_{f} \Gamma_{f \bar{f}}
$$

In the following $\Gamma_{h a d}=\sum_{q} \Gamma_{q \bar{q}}$ is the hadronic decay width of the $Z$ boson.

The hadronic peak cross section is defined as

$$
\sigma_{h}=\frac{12 \pi}{M_{Z}^{2}} \frac{\Gamma_{e e} \Gamma_{h a d}}{\Gamma_{Z}^{2}}
$$

The ratio of the hadronic to the electronic decay width is defined as

$$
R_{e}=\frac{\Gamma_{h a d}}{\Gamma_{e e}}
$$

The ratio of the partial decay width for $Z \rightarrow b \bar{b}(c \bar{c})$ to the total hadronic decay width is given by

$$
R_{b(c)}=\frac{\Gamma_{b \bar{b}(c \bar{c})}}{\Gamma_{h a d}} .
$$

The following quantities and observables depend on the ratio of the vector to axial vector coupling. The effective flavour dependent weak mixing angle can be written as

$$
\sin ^{2} \theta_{e f f}^{f}=\frac{1}{4\left|Q_{f}\right|}\left(1-\frac{v_{e f f}^{f}}{a_{e f f}^{f}}\right) .
$$

The left-right asymmetries are given by

$$
A_{L R}^{f}=\mathcal{A}^{\mathrm{f}}=\frac{2 v_{e f f}^{f} / a_{e f f}^{f}}{1+\left(v_{\text {eff }}^{f} / a_{\text {eff }}^{f}\right)^{2}},
$$

while the forward-backward asymmetries can be written as

$$
A_{F B}^{f}=\frac{3}{4} \mathcal{A}^{\mathrm{e}} \mathcal{A}^{\mathrm{f}}
$$




\section{The MSSM}

\subsection{Higgs sector}

The scalar sector of the MSSM is completely determined by the value of $\tan \beta=$ $v_{2} / v_{1}$ and the pseudoscalar mass $M_{A}$, together with the radiative corrections. The latter ones are taken into account in terms of the effective potential approximation with the leading terms $\sim m_{t}^{4}$, including the mixing in the scalar top system [31]. In this way, the coupling constants of the various Higgs particles to gauge bosons and fermions can be taken over from [30] substituting only the scalar mixing angle $\alpha$ by the improved effective mixing angle which is obtained from the diagonalization of the scalar mass matrix.

\subsection{Sfermion sector}

The physical masses of squarks and sleptons are described by a $2 \times 2$ mass matrix:

$$
\mathcal{M}_{\tilde{\mathrm{f}}}^{2}=\left(\begin{array}{ll}
M_{\tilde{\mathrm{Q}}}^{2}+m_{f}^{2}+M_{Z}^{2}\left(I_{3}^{f}-Q_{f} s_{W}^{2}\right) \cos 2 \beta & m_{f}\left(A_{f}+\mu\{\cot \beta, \tan \beta\}\right) \\
m_{f}\left(A_{f}+\mu\{\cot \beta, \tan \beta\}\right) & M_{\{\tilde{U}, \tilde{D}\}}^{2}+m_{f}^{2}+M_{Z}^{2} Q_{f} s_{W}^{2} \cos 2 \beta
\end{array}\right),
$$

with SUSY soft breaking parameters $M_{\tilde{Q}}, M_{\tilde{U}}, M_{\tilde{D}}, A_{f}$, and $\mu$. It is convenient to use the following notation for the off-diagonal entries in eq. (21):

$$
A_{f}^{\prime}=A_{f}+\mu\{\cot \beta, \tan \beta\} .
$$

Scalar neutrinos appear only as left-handed mass eigenstates. Up and down type sfermions in (21) are distinguished by setting $\mathrm{f}=\mathrm{u}, \mathrm{d}$ and the $\{u, d\}$ entries in the parenthesis. Since the non-diagonal terms are proportional to $m_{f}$, it seems natural to assume unmixed sfermions for the lepton and quark case except for the scalar top sector. The $\tilde{t}$ mass matrix is diagonalized by a rotation matrix with a mixing angle $\Phi_{m i x}$. Instead of $M_{\tilde{Q}}, M_{\tilde{U}}, M_{\tilde{D}}, A_{t}^{\prime}$ for the $\tilde{b}, \tilde{t}$ system the physical squark masses $m_{\tilde{b}_{L}}, m_{\tilde{b}_{R}}, m_{\tilde{t}_{2}}$ can be used together with $A_{t}^{\prime}$ or, alternatively, the stop mixing angle $\Phi_{m i x}$. For simplicity we assume $m_{\tilde{b}_{L}}=m_{\tilde{b}_{R}}$, and $\tilde{u}, \tilde{d}, \tilde{c}, \tilde{s}$ to have masses masses equal to the $\tilde{b}$ squark mass.

A possible mass splitting between $\tilde{b}_{L}-\tilde{t}_{L}$ yields a contribution to the $\rho$-parameter $\rho=1+\Delta \rho$ in terms of [22]: $\|$

$$
\Delta \rho_{\tilde{b}-\tilde{t}}^{0}=\frac{3 \alpha_{E M}}{16 \pi s_{W}^{2} M_{W}^{2}}\left(m_{\tilde{b}_{L}}^{2}+m_{\tilde{t}_{L}}^{2}-2 \frac{m_{\tilde{b}_{L}}^{2} m_{\tilde{t}_{L}}^{2}}{m_{\tilde{b}_{L}}^{2}-m_{\tilde{t}_{L}}^{2}} \log \frac{m_{\tilde{b}_{L}}^{2}}{m_{\tilde{t}_{L}}^{2}}\right) .
$$

\footnotetext{
${ }$ The superscript $\Delta \rho^{0}$ indicates that no left-right mixing is present.
} 
As a universal loop contribution, it enters the quantity

$$
\Delta r \simeq \Delta \alpha_{E M}-\frac{c_{W}^{2}}{s_{W}^{2}} \Delta \rho+\ldots
$$

and all the $Z$ boson widths

$$
\Gamma_{f \bar{f}} \sim 1+\Delta \rho+\cdots
$$

and is thus significantly constrained by the data on $M_{W}$ and the leptonic widths.

\subsection{Chargino/Neutralino sector}

The chargino (neutralino) masses and the mixing angles in the gaugino couplings are calculated from soft breaking parameters $M_{1}, M_{2}$ and $\mu$ in the chargino (neutralino) mass matrix[30]. The validity of the GUT relation $M_{1}=5 / 3 \tan ^{2} \theta_{W} M_{2}$ is assumed.

The chargino $2 \times 2$ mass matrix is given by

$$
\mathcal{M}_{\tilde{\chi}^{ \pm}}=\left(\begin{array}{ll}
M_{2} & M_{W} \sqrt{2} \sin \beta \\
M_{W} \sqrt{2} \cos \beta & -\mu
\end{array}\right),
$$

with the SUSY soft breaking parameters $\mu$ and $M_{2}$ in the diagonal matrix elements. The physical chargino mass states $\tilde{\chi}_{i}^{ \pm}$are the rotated wino and charged Higgsino states:

$$
\begin{aligned}
& \tilde{\chi}_{i}^{+}=V_{i j} \psi_{j}^{+} \\
& \tilde{\chi}_{i}^{-}=U_{i j} \psi_{j}^{-} ; i, j=1,2 .
\end{aligned}
$$

$V_{i j}$ and $U_{i j}$ are unitary chargino mixing matrices obtained from the diagonalization of the mass matrix eq. 25:

$$
\mathrm{U}^{*} \mathcal{M}_{\tilde{\chi}^{ \pm}} \mathrm{V}^{-1}=\operatorname{diag}\left(\mathrm{m}_{\tilde{\chi}_{1}^{ \pm}}, \mathrm{m}_{\tilde{\chi}_{2}^{ \pm}}\right)
$$

The neutralino $4 \times 4$ mass matrix can be written as:

$$
\mathcal{M}_{\tilde{\chi}^{0}}=\left(\begin{array}{cccc}
M_{1} & 0 & -M_{Z} \sin \theta_{W} \cos \beta & M_{Z} \sin \theta_{W} \sin \beta \\
0 & M_{2} & M_{Z} \cos \theta_{W} \cos \beta & -M_{Z} \cos \theta_{W} \sin \beta \\
-M_{Z} \sin \theta_{W} \cos \beta & M_{Z} \cos \theta_{W} \cos \beta & 0 & \mu \\
M_{Z} \sin \theta_{W} \sin \beta & -M_{Z} \cos \theta_{W} \sin \beta & \mu & 0
\end{array}\right)
$$

where the diagonalization can be obtained by the unitary matrix $N_{i j}$ :

$$
\mathrm{N}^{*} \mathcal{M}_{\tilde{\chi}^{0}} \mathrm{~N}^{-1}=\operatorname{diag}\left(\mathrm{m}_{\tilde{\chi}_{\mathrm{i}}^{0}}\right)
$$

The elements $U_{i j}, V_{i j}, N_{i j}$ of the diagonalization matrices enter the couplings of the charginos, neutralinos and sfermions to fermions and gauge bosons, as explicitly given 
in ref. [30]. Note that our sign convention on the parameter $\mu$ is opposite to that of ref. [30.

\section{Results}

\subsection{Chargino Masses}

As mentioned before the low $\tan \beta$ scenario of the MSSM needs a light right handed stop and light higgsino-like chargino for a large value of $R_{b}$, whereas in the high $\tan \beta$ scenario one needs in addition a light pseudoscalar Higgs $A$ [1, 3]. A higgsino - like chargino can be obtained for a low value of the parameter $\mu$ in the mass matrix (eq. 25). Figs. 1 and 5 show the dependence of the chargino masses on the parameter $\mu$ in a region of the parameter space which yields a good global $\chi^{2}$. In case of high $\tan \beta$, $m_{\tilde{\chi}_{2}}$ is almost symmetric around zero, whereas in case of low $\tan \beta$ this dependence is more complicated, as can be seen from fig. 1 . For $M_{2}=3|\mu|$ the light chargino mass passes zero at $\mu=-40$, so the following low $\tan \beta$ plots were made for $\mu>-40$ and $\mu \leq-40 \mathrm{GeV}$. The asymmetric structure of fig. $\forall$ is reflected in the contours of constant $R_{b}$ in the $m_{\tilde{\chi}_{2}}$ versus light scalar top $m_{\tilde{t}_{2}}$ plane (see fig. 6). High values of $R_{b}$ up to 0.2194 are possible (see figs. 6 and 7), although these special regions of the parameter space are already experimentally excluded by the lower limits on sparticle masses.

Taking $M_{2}=|\mu|$ does not change these results very much, as can be seen from a comparison of the $\chi^{2}$ distributions in fig. $8\left(M_{2}=3|\mu|\right)$ and fig. $9\left(M_{2}=|\mu|\right)$. The small increase of the $\chi^{2}$ at chargino masses around $80 \mathrm{GeV}$ in the left hand part of fig. 9 is due to neutralino threshold singularities, for which an additional $\chi^{2}$ contribution has been added, if the sum of two neutralino masses is close to the $Z^{0}$ mass. The sharp increase of the $\chi^{2}$ function at low chargino masses is due to experimental limits on chargino, neutralino and stop masses from LEP 1.5 [16, 17, 18].

\subsection{Optimization of Parameters}

An optimization of free parameters of the MSSM was performed by minimizing

a $\chi^{2}$ function using MINUIT [10]. Several contributions to the $\chi^{2}$ were taken into account:

- experimental limits on the masses of supersymmetric particles and neutralino production from LEP 1.5 and Tevatron [16, 17, 18]

- precision measurements of on resonance observables from LEP [14], taking error correlations into account

- the measurement of the branching ratio $\frac{B R(b \rightarrow s \gamma)}{B R(b \rightarrow c e \bar{\nu})}$ from CLEO [15] 


\begin{tabular}{|c|c|}
\hline \hline \multicolumn{2}{|c|}{ experimental limits } \\
\hline \hline$\tilde{m}_{\chi_{1,2}^{ \pm}}$ & $>65 \mathrm{GeV}$ \\
\hline$\tilde{m}_{\chi_{1}^{0}}$ & $>13 \mathrm{GeV}$ \\
$\tilde{m}_{\chi_{2}^{0}}$ & $>35 \mathrm{GeV}$ \\
$\tilde{m}_{\chi_{3,4}^{0}}$ & $>60 \mathrm{GeV}$ \\
$\Gamma_{Z \rightarrow \text { neutralinos }}$ & $<2 \mathrm{MeV}$ \\
\hline$\tilde{m}_{t_{1,2}}$ & $>48 \mathrm{GeV}$ \\
\hline$m_{h}, m_{H}, m_{A}, m_{H^{ \pm}}$ & $>50 \mathrm{GeV}$ \\
\hline
\end{tabular}

Table 1: Mass limits assumed for the optimized fits.

The experimental limits included in the fit are summarized in table 11 11, 16, 17, 18, 20. The calculation of the total decay width of the $\mathrm{Z}$ boson into neutralinos is based on reference [32], the calculation of the ratio $b \rightarrow s \gamma$ on reference [33].

As already mentioned, $R_{b}$ mainly depends on the stop mass and the light chargino mass for the low $\tan \beta$ and on the pseudoscalar Higgs mass and chargino mass for the high $\tan \beta$ scenario. In order to get a feeling for the size of the effects, we study the dependence of the $\chi^{2}$ function on these parameters with optimization of the remaining parameters. This has been done for both the low and the high $\tan \beta$ solutions. The best solutions will be presented in the next chapter.

- Low $\tan \beta$ :

Fig. 10 shows the change in the best obtainable $\chi^{2}$ in the chargino - stop plane. For each value of the lighter scalar top $m_{\tilde{t}_{2}}$ and $m_{\tilde{\chi}_{2}^{ \pm}}$in a grid of $10 \times 10$ points an optimization of $m_{t}, \alpha_{s}$ and the stop mixing angle $\Phi_{m i x}$ was performed, assuming $M_{2}=3|\mu|$ for a fixed value of $\tan \beta=1.6$. In the next section this assumption will be dropped. Low sparticle masses yield a sharp increase in the $\Delta \chi^{2}$ in fig. 10 because of the included mass limits. The minimum $\chi^{2}$ is obtained for chargino masses just above the experimental limit, although it increases only slowly with increasing sparticle masses. $R_{b}$ increases significantly with decreasing values of the stop and chargino mass, as can be seen from fig. 11. Much less significant is the improvement of $R_{c}$. Within the plane of fig. 11 it changes less than 0.0005 units. The increase of $R_{b}$ must be compensated by a decrease of $\alpha_{s}$ (see fig. 12) in order to keep the total Z-width constant. The stop mixing angle $\Phi_{m i x}$, shown in fig. 13, is mainly determined by the CLEO measurement of $b \rightarrow s \gamma$. The chargino contribution to $b \rightarrow s \gamma$ is proportional to the Higgs mixing parameter $\mu$, which changes its sign for $m_{\tilde{\chi}^{ \pm}} \approx 60 \mathrm{GeV}$ (see fig.(1), so the $b \rightarrow s \gamma$ rate changes rapidly for these chargino masses, as shown in fig. 14. The uncertainty in the predicted $b \rightarrow s \gamma$ rate from the renormalization scale 
has been taken into account and was varied between $m_{b} / 2$ and $2 m_{b}$ [34]. The scale itself has been chosen to be $m_{b}$.

- High $\tan \beta$ :

Similar fits can be performed for the high $\tan \beta$ scenario in the pseudo scalar Higgs $m_{A}$ versus light chargino plane. As in the low $\tan \beta$ case $M_{2}=3|\mu|$ was assumed. In fig. 15 the resulting change in the $\chi^{2}$ is given for fixed $\tan \beta=$ 50. For small chargino masses there is a sharp increase in the $\chi^{2}$ due to the corresponding mass limit, see above. The best values for $R_{b}$ can be obtained for small values of $m_{A}$ and $m_{\tilde{\chi}^{ \pm}}$, see fig. 16. As in the low $\tan \beta$ case the enhancement of $R_{b}$ must be compensated by a decrease of $\alpha_{s}$, see fig. 17, and the improvement of $R_{c}$ is small, less than 0.0006 within the given parameter plane. The mixing angle,shown in fig. 18, is mainly determined by the $b \rightarrow s \gamma$ rate, which can be fitted in the whole $m_{A}$ - chargino plane, see fig. 19 .

\subsection{Best Solutions}

Standard Model Fits:

The input values from $M_{W}, m_{t}$, the electroweak mixing angle and the $Z^{0}$ line shape observables have been summarized in table 2. The SM predictions were obtained from the ZFITTER package [35] and all the error correlations were taken from [14]. The fits were made with $\alpha_{s}, m_{t}$ and $m_{H}$ as free parameters, which resulted in

$$
\begin{gathered}
\alpha_{s}=0.1215 \pm 0.0036 \\
m_{t}=167.3_{-7.6}^{+8.2} \mathrm{GeV} \\
m_{H}=66_{-37}^{+81} \mathrm{GeV} .
\end{gathered}
$$

The quoted errors have been determined using MINOS [10]. Further details of the procedure are described elsewhere, see for example [36, 37, 38]. The $\chi^{2} /$ d.o.f of the $\mathrm{SM}$ fit is $23.2 / 15$ which corresponds to a probability of $8 \%$. Here, the main contributions to the $\chi^{2}$ originate from $R_{b}\left(\Delta \chi^{2}=10.7\right), \sin ^{2} \Theta_{\text {eff }}^{\text {lept }}$ from $\operatorname{SLD}\left(\Delta \chi^{2}=3.6\right)$ and $R_{c}\left(\Delta \chi^{2}=3.3\right)$. The correlation parameter between $m_{H}$ and $m_{t}$ for the best fit is approximately 0.7 ; this strong correlation is shown in fig. 20. One observes that the upper limit on the Higgs mass is obtained for $m_{t} \approx 175 \mathrm{GeV}$; however, the upper limit is sensitive to $\sin ^{2} \Theta_{\text {eff }}^{\text {lept }}$ as shown by the dashed contour in fig. 20, where the precise value of $\sin ^{2} \Theta_{\text {eff }}^{\text {lept }}$ from SLD was excluded from the fit. The dependence of $\sin ^{2} \Theta_{\text {eff }}^{\text {lept }}$ on the SM Higgs mass is approximately logarithmic (see fig. 21). The LEP data alone without SLD yields $m_{H}=144_{-82}^{+164} \mathrm{GeV}$, SLD alone yields $m_{H}=15_{-8}^{+25} \mathrm{GeV}$, as indicated by the squares in fig. 21. The latter value is excluded by the lower 
limit of $58.4 \mathrm{GeV}$ from the combined LEP experiments [20]. In addition, the ALEPH Collaboration gives a recent limit of $63.9 \mathrm{GeV}$ on the SM Higgs mass [19]. The $\Delta \chi^{2}$ dependence of the Higgs mass is shown in fig. 22 for various conditions.

\section{MSSM Fits and Comparison with the SM:}

In order to obtain the best MSSM fits the assumption $M_{2}=3|\mu|$ is dropped and $M_{2}$ is treated as a free parameter. $R_{b}$ increases with decreasing $\tan \beta$. The fit results for a $\tan \beta$ as low as one are given in the first column of table 3 , the corresponding predictions of electroweak observables in table 2. Note the high value of $R_{b}$ and the corresponding low value of $\alpha_{s}$. The resulting $\chi^{2} /$ d.o.f. is $15.1 / 11$, corresponding to a probability of about $18 \%$. Unfortunately, the corresponding $b \rightarrow s \gamma$ rate is about one order of magnitude too small in this region of parameter space. Larger rates can be obtained either by heavier sparticle masses or by larger values of $\tan \beta$. With $b \rightarrow s \gamma$ included in the fit and a free $\tan \beta$, the preferred value of $\tan \beta$ was either around 1.6 or 50 .

The fit results for these values of $\tan \beta$ are given in table 3 too and the predicted values of all observables with their pulls have been summarized in table 2. Note that the MSSM prediction of the W-boson mass is always higher than the Standard Model one.

For the best solutions the gluino mass was fixed to $1500 \mathrm{GeV}$, the stau mass to $500 \mathrm{GeV}$ and the sbottom mass to $1000 \mathrm{GeV}$ in both the low and the high $\tan \beta$ scenario, since they are less sensitive to the LEP observables and therefore cannot be fitted. Their influence was studied by fixing them to different values and repeating the fits again. First the low $\tan \beta$ scenario will be discussed. A variation of the gluino mass from $200 \mathrm{GeV}$ up to $2000 \mathrm{GeV}$ did not change the best obtainable $\chi^{2}$, varying the stau mass in the same range changed the $\chi^{2}$ less than 0.2 . Furthermore, the best obtainable $\chi^{2}$ changed less than 0.2 when varying the sbottom mass and pseudoscalar Higgs mass from $800 \mathrm{GeV}$ to $2000 \mathrm{GeV}$. For values of these two parameters below $800 \mathrm{GeV}$ the $\chi^{2}$ increased significantly, mainly because the prediction of $R_{b}$ became too small.

Within the high $\tan \beta$ scenario no significant change of the global $\chi^{2}$ was detected when the gluino mass was varied between $200 \mathrm{GeV}$ and $2000 \mathrm{GeV}$, but the stau mass was somewhat more sensitive. A variation of this parameter between $300 \mathrm{GeV}$ and $700 \mathrm{GeV}$ changed the global $\chi^{2}$ less than 0.2 , but if it was chosen higher than $1000 \mathrm{GeV}$ the $\chi^{2}$ increased up to 1.6 units, mainly because the prediction of $\mathcal{A}_{\tau}$ became worse. The sbottom mass was varied between $800 \mathrm{GeV}$ and $2000 \mathrm{GeV}$. As in the low $\tan \beta$ case there was no dependence on this parameter if it was chosen heavy, but for low values the preferred top mass became too small. $M_{2}$ was fixed at $1500 \mathrm{GeV}$. A variation between $1000 \mathrm{GeV}$ and $2000 \mathrm{GeV}$ changed the best reachable $\chi^{2}$ less than 0.2 , for smaller values the global $\chi^{2}$ increased up to one unit.

To check the influence of the assumptions on $M_{1}$ for the best fits, the GUT relation 
$M_{1}=5 / 3 \tan ^{2} \theta_{W} M_{2}$ was dropped and the fit was repeated with a free $M_{1}$, but this did not improve the best obtainable $\chi^{2}$ significantly.

A direct comparison to the Standard Model fits is given for all three fits in figs. 24 26. The resulting Standard Model $\chi^{2} /$ d.o.f. $=23.2 / 15$ corresponds to a probability of $8 \%$, the MSSM fits correspond to probabilities of $15 \%(\tan \beta=1.6$, $\chi^{2} /$ d.o.f. $\left.=16.9 / 12\right)$ and $10 \%\left(\tan \beta=50, \chi^{2} /\right.$ d.o.f. $\left.=18.4 / 12\right)$. In counting the d.o.f the insensitive (and fixed) parameters were ignored. The high $\tan \beta$ scenario is hardly more probable than the SM, while for low $\tan \beta$ the probability of $15 \%$ is in between the best MSSM fit without $b \rightarrow s \gamma$ and the SM fit.

Another interesting point are the predictions for $\alpha_{s}\left(M_{Z}\right)$. Fig. 27 shows a comparison of different measurements of $\alpha_{s}\left(M_{Z}\right)$ (as given in [20, 39]) with the fitted values given in this paper. The fitted MSSM $\alpha_{s}\left(M_{Z}\right)$ is slightly smaller than the Standard Model value and in better agreement with measurements from deep inelastic lepton scattering (DIS) and the world average[20].

\subsection{Discovery Potential at LEP II}

The particle spectrum for the best fits, as shown in table 3, suggests that some SUSY particles could be within reach of LEP II. If they are not found, LEP II will provide stringent SUSY mass limits 37, 38. In the following the consequences of increased mass limits on the fits are discussed for both the low and high $\tan \beta$ scenario.

\section{Chargino Searches:}

The $\chi^{2}$ in the region of the best low $\tan \beta$ fit increases slowly for increasing chargino masses, see fig. 23. Chargino masses above a possible LEP II limit of $95 \mathrm{GeV}$ will increase the global $\chi^{2}$ of the fit by approximately 2 units, which corresponds to a probability of $9 \%$, which is hardly better than the SM probability, so one cannot consider the MSSM as a better solution in that case. For the high $\tan \beta$ scenario the dependence of the global $\chi^{2}$ on the chargino mass is small, see fig.15, so increased limits will hardly change the probability of about $10 \%$ for the best possible fit in this scenario.

\section{Stop and Neutralino Searches:}

The best $\tan \beta=1.6$ fit has a light stop mass of about $48 \mathrm{GeV}$, and the lightest neutralino is about $20 \mathrm{GeV}$, so this solution is just in between the regions of the parameter space which are excluded by stop searches at LEP1 and the Tevatron [18]: LEP I limits for light right handed stops are about $45 \mathrm{GeV}$, while D0 limits exclude $52(70) \mathrm{GeV}<m_{\tilde{t}_{2}}<92(87) \mathrm{GeV}$ for $m_{\tilde{\chi}_{0}}=20$ (40) GeV. A fit with increased neutralino mass limits of $45 \mathrm{GeV}$ yielded a best solution of $\chi^{2} /$ d.o.f. $=17.8 / 12$, corresponding to a probability of $12 \%$, which is worse than the solution presented 
above, but still better than the Standard Model one. A similar $\chi^{2} /$ d.o.f. was obtained if the light stop was required to be heavier than $90 \mathrm{GeV}$. The reason for this can be found in the flat $\chi^{2}$ distribution in fig. 23. This figure is similar to fig. 10, but here $M_{2}$ was treated as a free input parameter.

The stop mass in the high $\tan \beta$ solution is $53 \mathrm{GeV}$, and the lightest neutralino is quite heavy, above $70 \mathrm{GeV}$, so there is no conflict with stop searches. Furthermore, the high $\tan \beta$ is insensitive to the stop mass, so an inreased mass limit does not effect the fit.

\section{Higgs Searches:}

The mass of the light scalar Higgs is a sensitive function of the top mass. If the top mass is below $180 \mathrm{GeV}$, the Higgs mass for the low $\tan \beta$ fit (see tab. 3) should be observable at LEP II, especially if one includes the second order corrections, which will lower the Higgs mass by $10-15 \mathrm{GeV}$ [38]. Within the high $\tan \beta$ scenario both neutral Higgs bosons are light and have practically the same mass. Increasing the Higgs limits up to $90 \mathrm{GeV}$ increases the $\chi^{2} /$ dof to $23.7 / 12$, corresponding to a probability of only $2 \%$, which is much worse than the SM fit. The steep dependence of the $\chi^{2}$ on the pseudoscalar Higgs mass, which is mainly caused by a too small value of $R_{b}$, is shown in fig. 15 .

\section{CMSSM and $R_{b}$}

In ref. [37] fits to low energy data have been performed within the constrained MSSM (CMSSM). In this case unification of gauge and b- $\tau$ Yukawa couplings is assumed. Reproducing the large mass splitting in the stop sector, as given in tab. 3, needs a very artificial fine tuning of the few free parameters of the CMSSM, especially for the trilinear and Yukawa coupling in the stop sector, which can drive the diagonal elements of the stop matrix, eq. 21, apart. Note that the off-diagonal elements of this matrix are too small to generate a large splitting, since the left-handed stop is considerably heavier than the top, implying that one of the diagonal elements is considerably larger than the off-diagonal elements.

In addition, problems arise with electroweak symmetry breaking, since this requires $\mu>M_{2}$, while $R_{b}$ requires $\mu<M_{2}$ for a significant improvement. In conclusion, within the CMSSM neither the high value of $R_{b}$ nor the low value of $R_{c}$ can be explained, so in this scenario the solution must be sought in common systematic errors for all experiments, like PDB branching ratios in the charm - sector 40 causing the too high value of $R_{b}$ and too low value of $R_{c}$.

\section{Conclusions}

The MSSM provides a good description of all electroweak data. $R_{b}$ values up to 
0.2193 are possible, but the $b \rightarrow s \gamma$ rate is too low in this case. With all mass bounds and the $b \rightarrow s \gamma$ rate included in the fit, the best $\chi^{2} /$ d.o. $f$ in the MSSM is $16.9 / 12$, which corresponds to a probability of $15 \%$ as compared to the SM probability of about $8 \%$. This best MSSM fit with light stops and light charginos is obtained for $\tan \beta=1$.6. Another solution with $\tan \beta=50$ and light Higgses has a probability of $10 \%$, which is not much of an improvement over the SM.

The enhancement of $R_{b}$ is compensated by a decrease in $\alpha_{s}\left(M_{Z}\right)$ from 0.1215 in the SM case to 0.116 in the MSSM. The latter is in somewhat better agreement with precise measurements from DIS at low energies $\left(\alpha_{s}\left(M_{Z}\right)=0.112 \pm 0.005\right)$.

The best solutions predict chargino, stop and pseudoscalar Higgs masses which may be detectable at LEP II. The high $\tan \beta$ solution, requiring light Higgses, can certainly be excluded at LEP II, if no Higgses are found. For the low $\tan \beta$ scenario it will be more difficult to exclude the SUSY explanation of the too high value of $R_{b}$, since even for chargino and stop masses above $95 \mathrm{GeV}$ moderate improvements are still possible. On the other hand, it has to be pointed out that the large splittings within the stop sector, which yield the best fit, are very difficult to obtain in a natural way within a constrained MSSM model, since they require a very special finetuning of the trilinear and Yukawa couplings. 


\begin{tabular}{|c|c|c|c|c|c|c|c|c|c|}
\hline \multirow{3}{*}{$\begin{array}{l}\text { Symbol } \\
\tan \beta \text { and pull }\end{array}$} & \multirow[t]{3}{*}{ measurement } & \multicolumn{8}{|c|}{ best fit observables } \\
\hline & & \multicolumn{2}{|l|}{$\overline{\mathrm{SM}}$} & \multicolumn{6}{|c|}{$\begin{array}{lc}\text { MSSM } \\
\end{array}$} \\
\hline & & & pull & 1.0 & pull & 1.6 & pull & 50 & pull \\
\hline 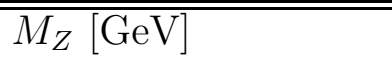 & 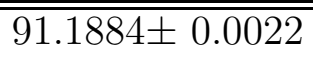 & 91.1882 & 0.089 & 91.1884 & 0.000 & 91.1884 & 0.000 & 91.1884 & 0.000 \\
\hline$\Gamma_{Z}[\mathrm{GeV}]$ & $2.4964 \pm 0.0032$ & 2.4973 & -0.284 & 2.4956 & 0.241 & 2.4958 & 0.202 & 2.4952 & 0.363 \\
\hline$\sigma_{h}[\mathrm{nb}]$ & $41.49 \pm 0.078$ & 41.452 & 0.483 & 41.441 & 0.634 & 41.448 & 0.540 & 41.429 & 0.777 \\
\hline$R_{l}$ & $20.789 \pm 0.032$ & 20.774 & 0.456 & 20.7878 & 0.037 & 20.7878 & 0.037 & 20.7898 & -0.024 \\
\hline$A_{F B}^{l}$ & $0.0171 \pm 0.0011$ & 0.0164 & 0.675 & 0.0165 & 0.577 & 0.0164 & 0.626 & 0.0165 & 0.505 \\
\hline$R_{b}$ & $0.2211 \pm 0.0016$ & 0.2159 & 3.222 & 0.2193 & 1.128 & 0.2180 & 1.915 & 0.2180 & 1.967 \\
\hline$R_{c}$ & $0.1598 \pm 0.0069$ & 0.1723 & -1.805 & 0.1703 & -1.522 & 0.1706 & -1.559 & 0.1706 & -1.565 \\
\hline$A_{F B}^{b}$ & $0.1002 \pm 0.0028$ & 0.1034 & -1.145 & 0.1040 & -1.366 & 0.1038 & -1.286 & 0.1043 & -1.453 \\
\hline$A_{F B}^{c}$ & $0.0759 \pm 0.0051$ & 0.0740 & 0.374 & 0.0743 & 0.319 & 0.0741 & 0.347 & 0.0745 & 0.281 \\
\hline $\mathcal{A}_{b}$ & $0.842 \pm 0.052$ & 0.9336 & -1.761 & 0.9361 & -1.810 & 0.9356 & -1.800 & 0.9361 & -1.809 \\
\hline $\mathcal{A}_{c}$ & $0.6180 \pm 0.091$ & 0.6680 & -0.550 & 0.6684 & -0.554 & 0.6682 & -0.552 & 0.6685 & -0.555 \\
\hline $\mathcal{A}_{\tau}$ & $0.1394 \pm 0.0069$ & 0.1477 & -1.196 & 0.1482 & -1.270 & 0.1479 & -1.235 & 0.1479 & -1.229 \\
\hline $\mathcal{A}_{e}$ & $0.1429 \pm 0.0079$ & 0.1477 & -0.605 & 0.1482 & -0.667 & 0.1479 & -0.636 & 0.1484 & -0.712 \\
\hline $\sin ^{2} \Theta_{e f f}^{\text {lept }}\left(\left\langle Q_{F B}\right\rangle\right)$ & $0.2320 \pm 0.0010$ & 0.2314 & 0.562 & 0.23138 & 0.624 & 0.23141 & 0.593 & 0.23133 & 0.669 \\
\hline$M_{W}[\mathrm{GeV}]$ & $80.33 \pm 0.15$ & 80.370 & -0.265 & 80.417 & -0.583 & 80.422 & -0.616 & 80.452 & -0.814 \\
\hline $1-M_{W}^{2} / M_{Z}^{2}$ & $0.2257 \pm 0.0047$ & 0.2232 & 0.531 & 0.2223 & 0.727 & 0.2222 & 0.747 & 0.2216 & 0.869 \\
\hline$m_{t}[\mathrm{GeV}]$ & $175 \pm 9$ & 167.3 & 0.858 & 172.8 & 0.239 & 172.1 & 0.32 & 168.0 & 0.776 \\
\hline $\sin ^{2} \Theta_{\text {eff }}^{l e p t}\left(A_{L R}\right)(\mathrm{SLD})$ & $0.23049 \pm 0.0005$ & 0.23144 & -1.900 & 0.23138 & -1.773 & 0.23141 & -1.834 & 0.23133 & -1.682 \\
\hline
\end{tabular}

Table 2: Measurements of the observables [14] and the predicted results of the fits with minimum $\chi^{2}$. The pulls are defined by (measurement - predicted value) / error of the measurement. 


\begin{tabular}{|c|c|c|c|}
\hline \multicolumn{4}{|c|}{ Fitted SUSY parameters and masses } \\
\hline Symbol & $\tan \beta=1.0$ & $\tan \beta=\mathbf{1 . 6}$ & $\tan \beta=50$ \\
\hline & no $b \rightarrow s \gamma$ & \multicolumn{2}{|c|}{$b \rightarrow s \gamma$ inc. } \\
\hline$m_{t}[\mathrm{GeV}]$ & $\begin{array}{rr}173 \pm 7 \\
0.1104 \pm 0.0043\end{array}$ & $\begin{array}{r}172 \pm 6 \\
0.1161 \pm 0.0038\end{array}$ & $\begin{array}{r}168 \pm 6 \\
0.1162 \pm 0.0039\end{array}$ \\
\hline$M_{2}[\mathrm{GeV}]$ & $25 \pm 8$ & $36 \pm 23$ & \\
\hline$\mu[\mathrm{GeV}]$ & $35 \pm 53$ & $42 \pm 9$ & $76 \pm 28$ \\
\hline$m_{\tilde{t}_{2}}[\mathrm{GeV}]$ & $48 \pm 5$ & $48 \pm 5$ & $53 \pm 40$ \\
\hline$\phi_{m i x}$ & $-0.163 \pm 0.115$ & $-0.203 \pm 0.091$ & $0.0021 \pm 0.0054$ \\
\hline$m_{A}[\mathrm{GeV}]$ & - & - & $50 \pm 5$ \\
\hline \multicolumn{4}{|c|}{ Particle Spectrum } \\
\hline$\overline{m_{\tilde{t}_{1}}}[\mathrm{GeV}]$ & \multicolumn{3}{|c|}{$\approx 1 \mathrm{TeV}$} \\
\hline$m_{\tilde{t}_{2}}[\mathrm{GeV}]$ & 48 & 48 & 53 \\
\hline$m_{\tilde{q}}[\mathrm{GeV}]$ & \multirow{2}{*}{\multicolumn{3}{|c|}{$\begin{array}{c}1 \mathrm{TeV} \\
0.5 \mathrm{TeV}\end{array}$}} \\
\hline$m_{\tilde{l}}[\mathrm{GeV}]$ & & & \\
\hline$m_{\tilde{\chi_{1}^{ \pm}}}[\mathrm{GeV}]$ & 91 & 106 & 1504 \\
\hline$m_{\tilde{\chi}_{2}^{ \pm}}^{\wedge}[\mathrm{GeV}]$ & 81 & 69 & 76 \\
\hline$m_{{\tilde{\chi_{0}^{0}}}_{0}}[\mathrm{GeV}]$ & 15 & 21 & 73 \\
\hline$m_{{\tilde{\chi_{2}^{0}}}_{1}^{\lambda_{1}}}[\mathrm{GeV}]$ & 35 & 38 & 79 \\
\hline$m_{\tilde{\chi_{3}^{0}}}^{\lambda}[\mathrm{GeV}]$ & 90 & 97 & 714 \\
\hline$m_{{\tilde{\chi_{4}^{0}}}_{0}^{0}}[\mathrm{GeV}]$ & 102 & 102 & 1504 \\
\hline$m_{h}[\mathrm{GeV}]$ & 97 & 110 & 50 \\
\hline$m_{H}[\mathrm{GeV}]$ & \multicolumn{2}{|c|}{$\approx 1.5 \mathrm{TeV}$} & 98 \\
\hline$m_{A}[\mathrm{GeV}]$ & \multicolumn{2}{|c|}{$1.5 \mathrm{TeV}$} & 50 \\
\hline$m_{H^{ \pm}}[\mathrm{GeV}]$ & \multicolumn{2}{|c|}{$\approx 1.5 \mathrm{TeV}$} & 143 \\
\hline$M_{W}^{ \pm}[\mathrm{GeV}]$ & 80.4174 & 80.4224 & 80.4520 \\
\hline$\frac{B R(b \rightarrow s \gamma)}{B R(b \rightarrow c e \bar{\nu})} / 10^{-4}$ & $(0.19)$ & 2.05 & 2.30 \\
\hline $\begin{array}{l}\chi^{2} / \text { d.o.f. } \\
\end{array}$ & $\overline{(15.1 / 11)}$ & 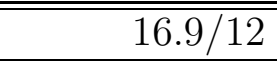 & 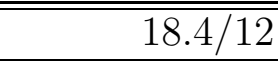 \\
\hline Probability & $18 \%$ & $15 \%$ & $10 \%$ \\
\hline
\end{tabular}

Table 3: Values of the fitted parameters (upper part) and corresponding mass spectrum (lower part). The errors on the parameters are parabolic ones. The parameters given in the first column gave a minimum $\chi^{2} /$ d.o.f. of 15.1/11 for the LEP - observables. Here $b \rightarrow s \gamma$ was not included in the fit, but the resulting $b \rightarrow s \gamma$ rate is about one order of magnitude too small. Including $b \rightarrow s \gamma$ rate as measured by the CLEO Collaboration [15] requires a higher value of $\tan \beta$, which reduces the best $\chi^{2} /$ d.o.f. to $16.9 / 12$ (second column). On the right hand side the results of the optimization for $\tan \beta=50$ are given. The dashes indicate irrelevant parameters which were chosen high. 


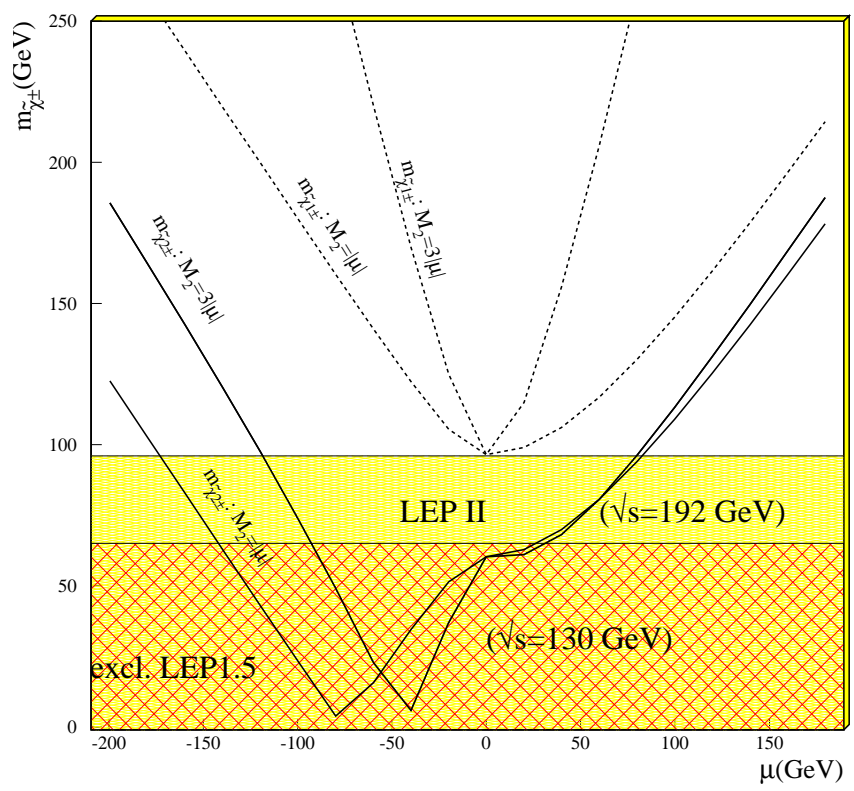

Figure 4: Dependence of the chargino masses on the parameter $\mu$ for $M_{2}=|\mu|$ and $M_{2}=3|\mu|$ for $\tan \beta=1.6, \alpha_{s} \approx 0.117$ and $\tilde{m}_{t_{2}} \approx 60 \mathrm{GeV}$. No optimization of parameters was performed here. The shaded regions indicate chargino masses less than $65 \mathrm{GeV}$ which are excluded by $L E P 1.5$ and chargino masses less than $96 \mathrm{GeV}$. It can be observed that for positive values of $\mu$ two light charginos are easier to obtain, if $\mu$ and $M_{2}$ have similar values.

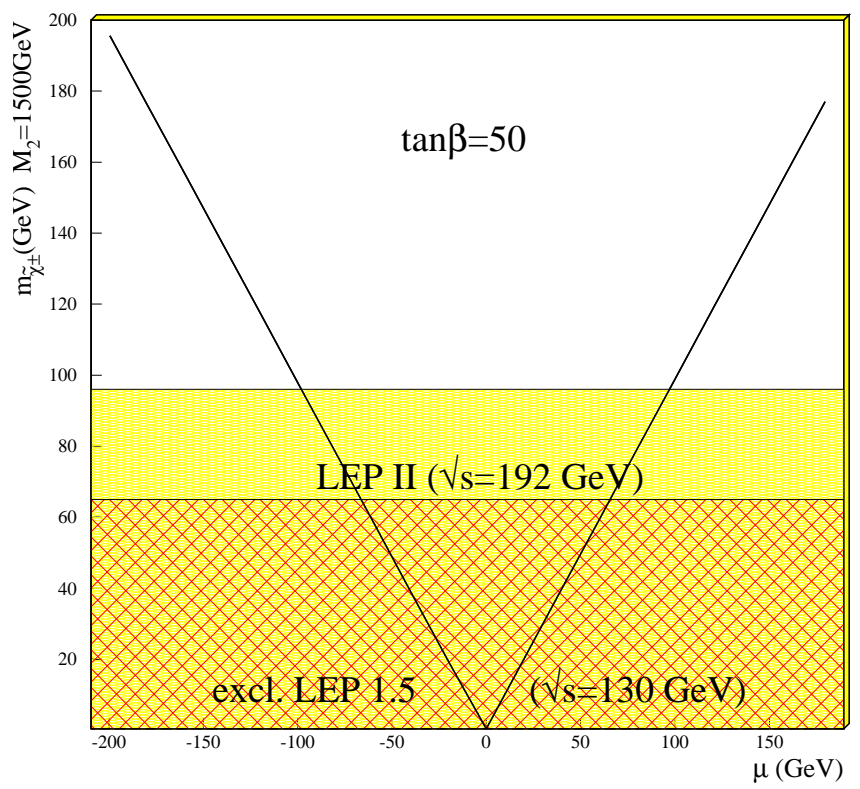

Figure 5: Dependence of the chargino masses on the parameter $\mu$ for $\tan \beta=50$. In this case $M_{2}=1500 \mathrm{GeV}$ was used and no optimization of parameters was performed. The shaded regions indicate chargino masses less than $65 \mathrm{GeV}$ which are excluded by LEP 1.5 and chargino masses less than $96 \mathrm{GeV}$. The light chargino mass depends on $\mu$, whereas the heavy chargino mass is dominated by the value of $M_{2}$ and keeps approximately constant at $1500 \mathrm{GeV}$. For a fixed given chargino mass there are two possible solutions corresponding to $\mu<0$ and $\mu>0$, respectively. 

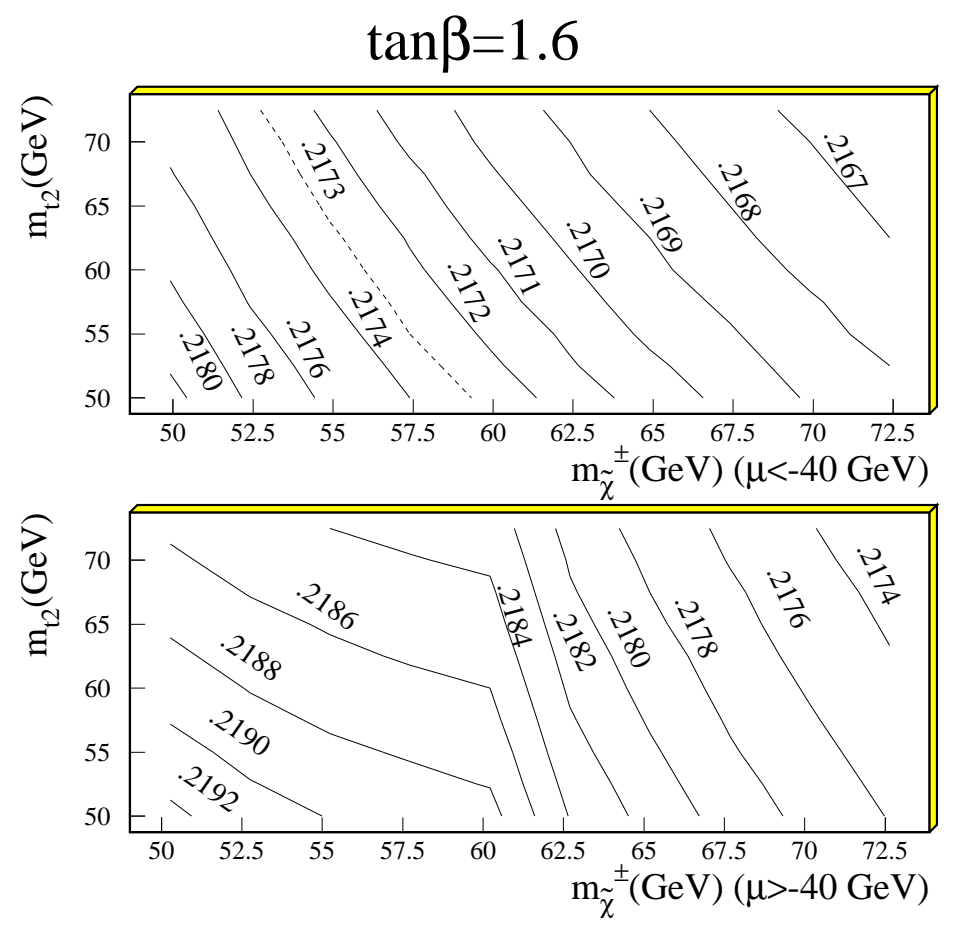

Figure 6: $\quad R_{b}$ in the light stop versus light chargino plane with $M_{2}=3|\mu|$ and $\tan \beta=1.6$. The upper part shows the solution with $\mu<-40 \mathrm{GeV}$, in the lower part the one with $\mu>-40 \mathrm{GeV}$ is displayed. In the latter solution quite high values for $R_{b}$ are possible, as can be seen in the figure. The dashed line in the upper plot indicates the $2 \sigma$ lower limit of $R_{b}$.

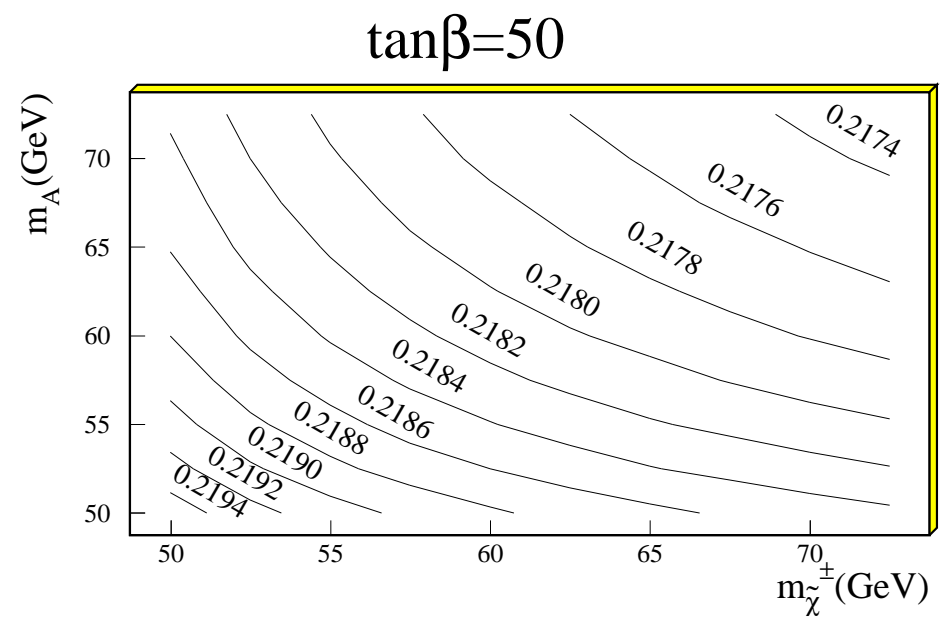

Figure 7: $\quad R_{b}$ in the $m_{A}$ versus light chargino plane with $M_{2}=3|\mu|$ for the high $\tan \beta$ solution. $\mu$ was chosen positive here. In this case choosing the opposite sign for $\mu$ doesn't change $R_{b}$. 

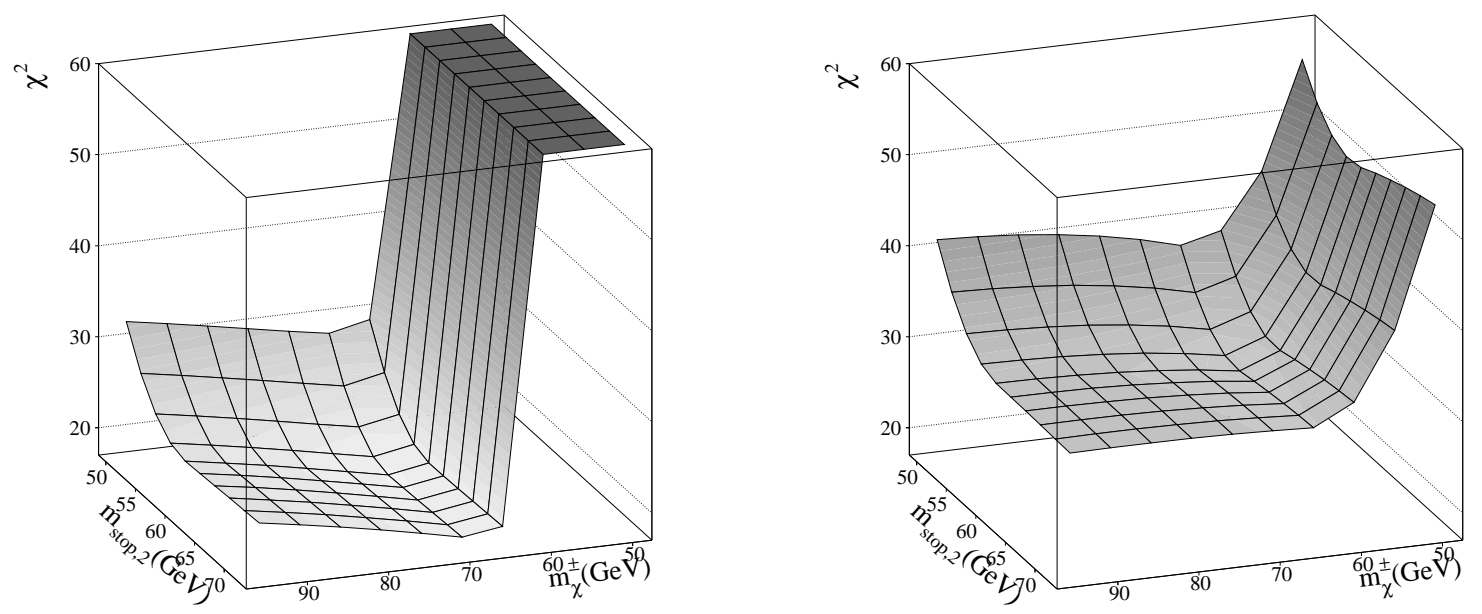

Figure 8: Dependence of the absolute $\chi^{2}$ for $\mu>-40 \mathrm{GeV}$ (left side) and $\mu<$ $-40 \mathrm{GeV}$ (right side), using $M_{2}=3|\mu|$. At the different points of the grid no optimisation of parameters was performed, but they were fixed to values near a good minimum.
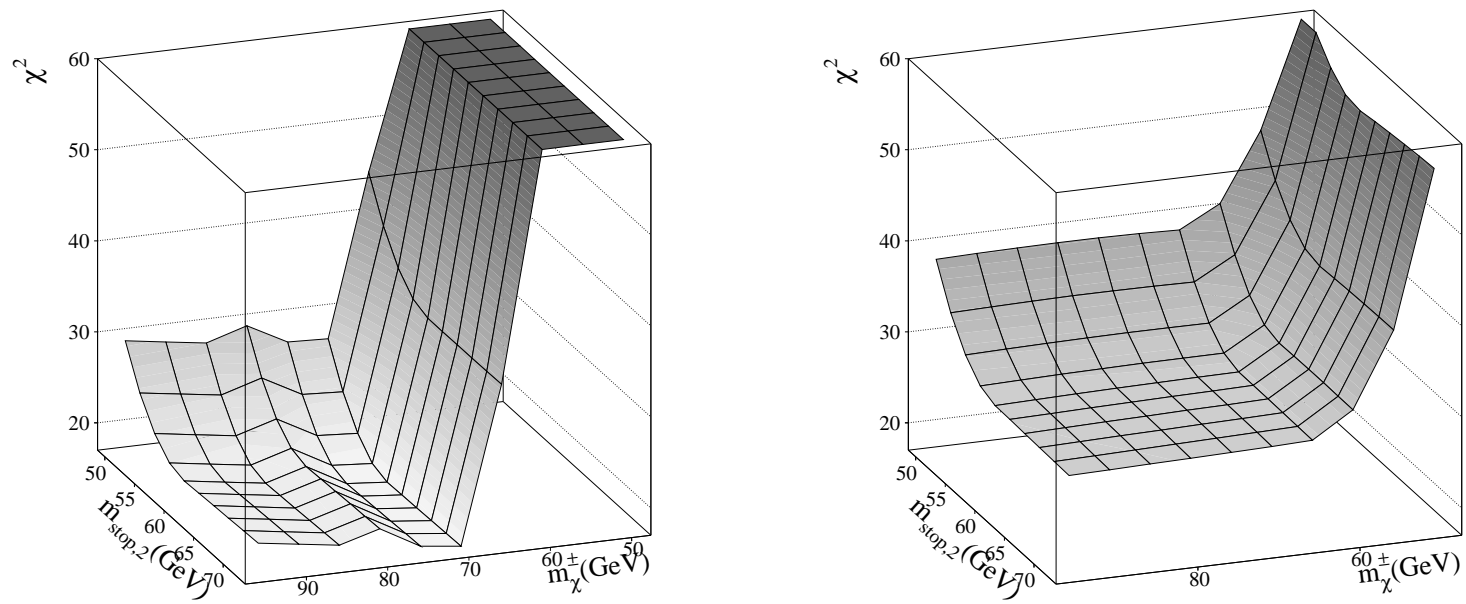

Figure 9: The same as 8, but for $M_{2}=|\mu|$. 


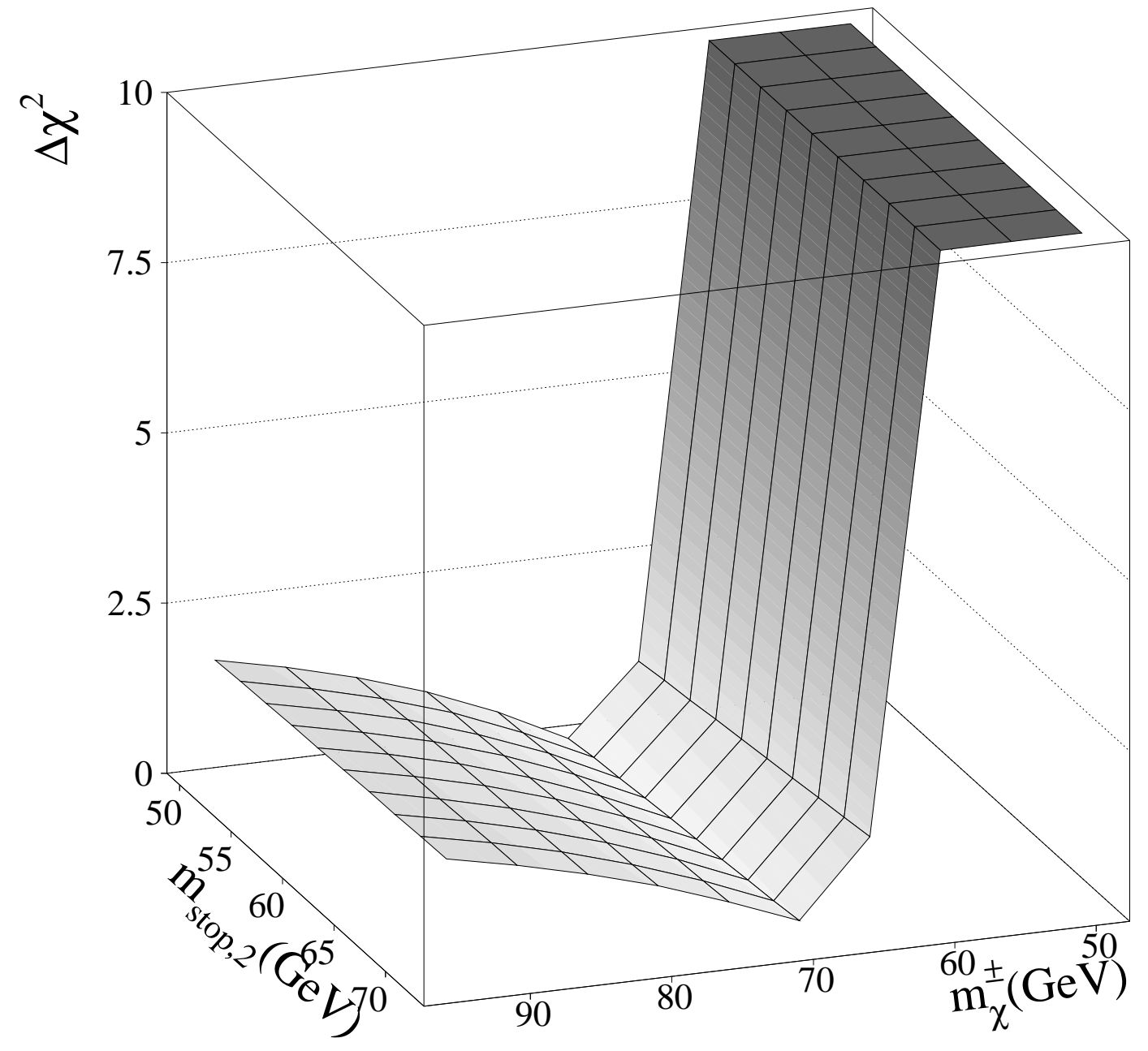

Figure 10: The $\Delta \chi^{2}$ in the light stop and light chargino plane for $\tan \beta=1.6$. At each point of the grid an optimization of $m_{t}, \alpha_{s}$ and the stop mixing angle $\phi_{\text {mix }}$ was performed with $\mu>-40$ and $M_{2}=3|\mu|$, including the ratio $b \rightarrow s \gamma$. 


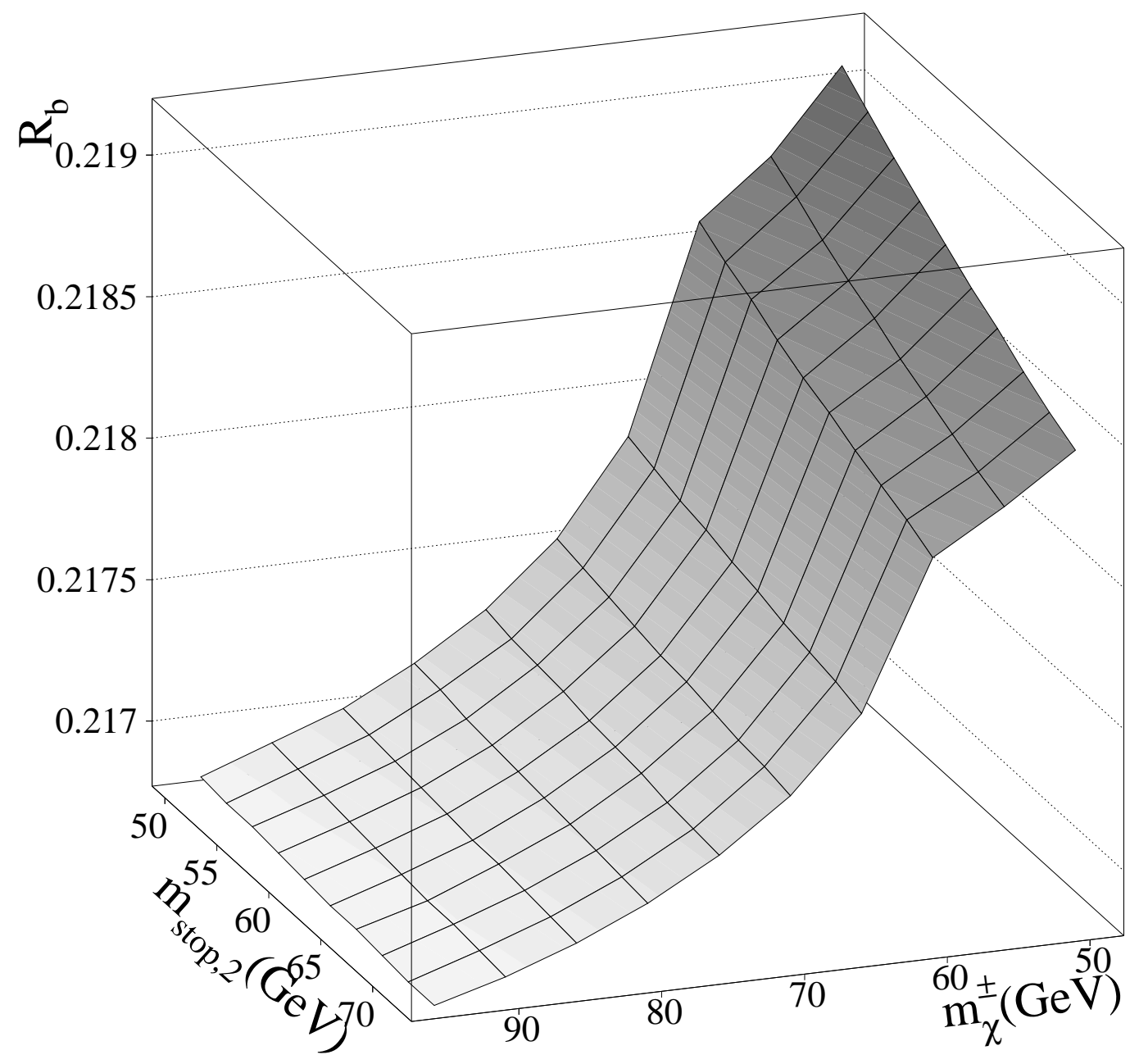

Figure 11: $R_{b}$ in the light stop and light chargino plane. Optimization as in fig. 10. 


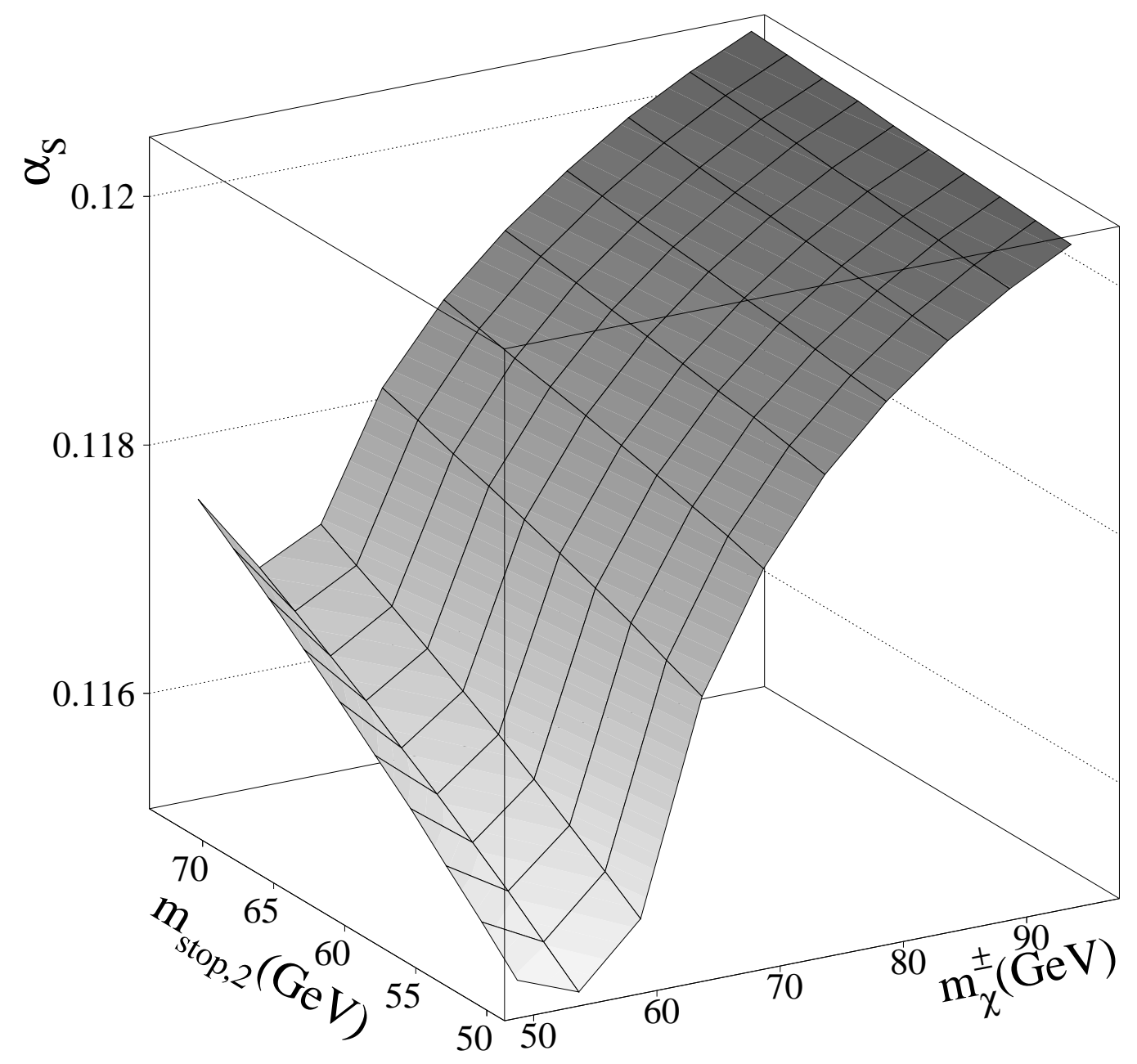

Figure 12: $\alpha_{s}$ in the light stop and light chargino plane. Optimization as in fig. 10. 


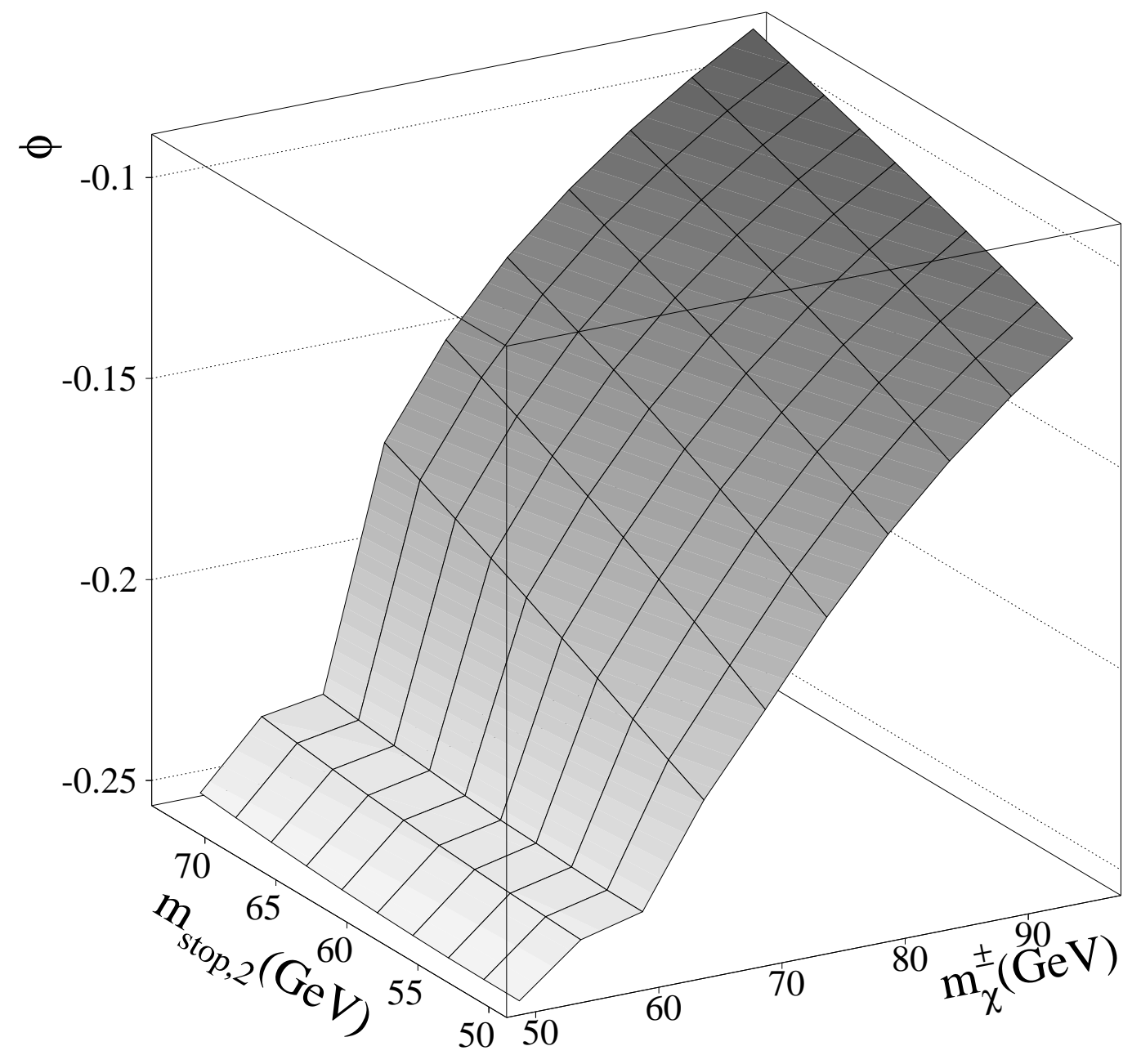

Figure 13: Stop mixing angle $\phi_{m i x}$ in the light stop and light chargino plane. It is mainly determined by the branching ratio $b \rightarrow s \gamma$. Optimization as in fig. 10 . 


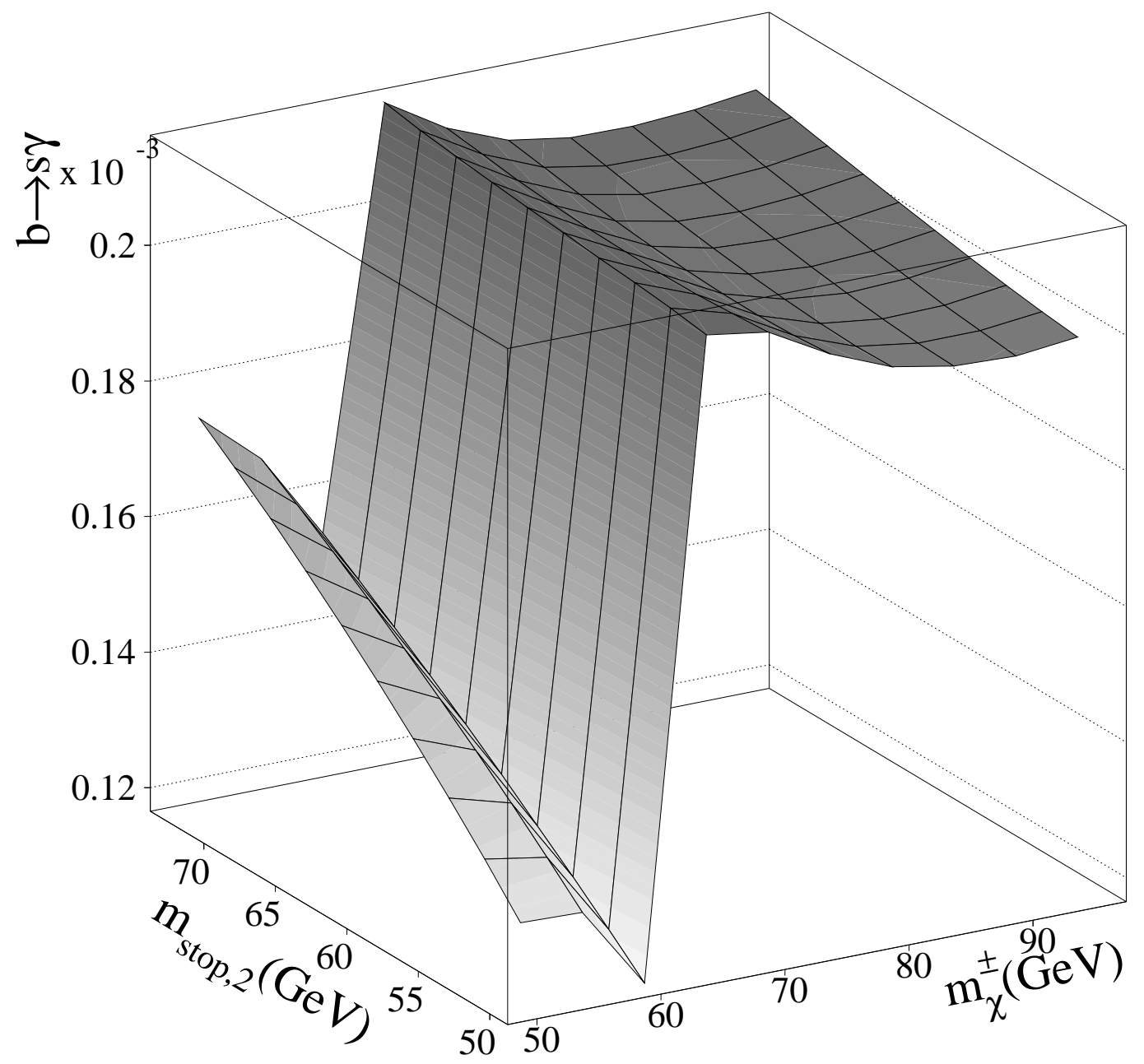

Figure 14: $\quad b \rightarrow s \gamma$ in the light stop and light chargino plane. For chargino masses higher than $60 \mathrm{GeV}$ (and $\mu>0$ ) the predicted value is close to the CLEO measurement of $2.32 \pm 0.67 \times 10^{-4}$. Optimization as in fig. 10 . 


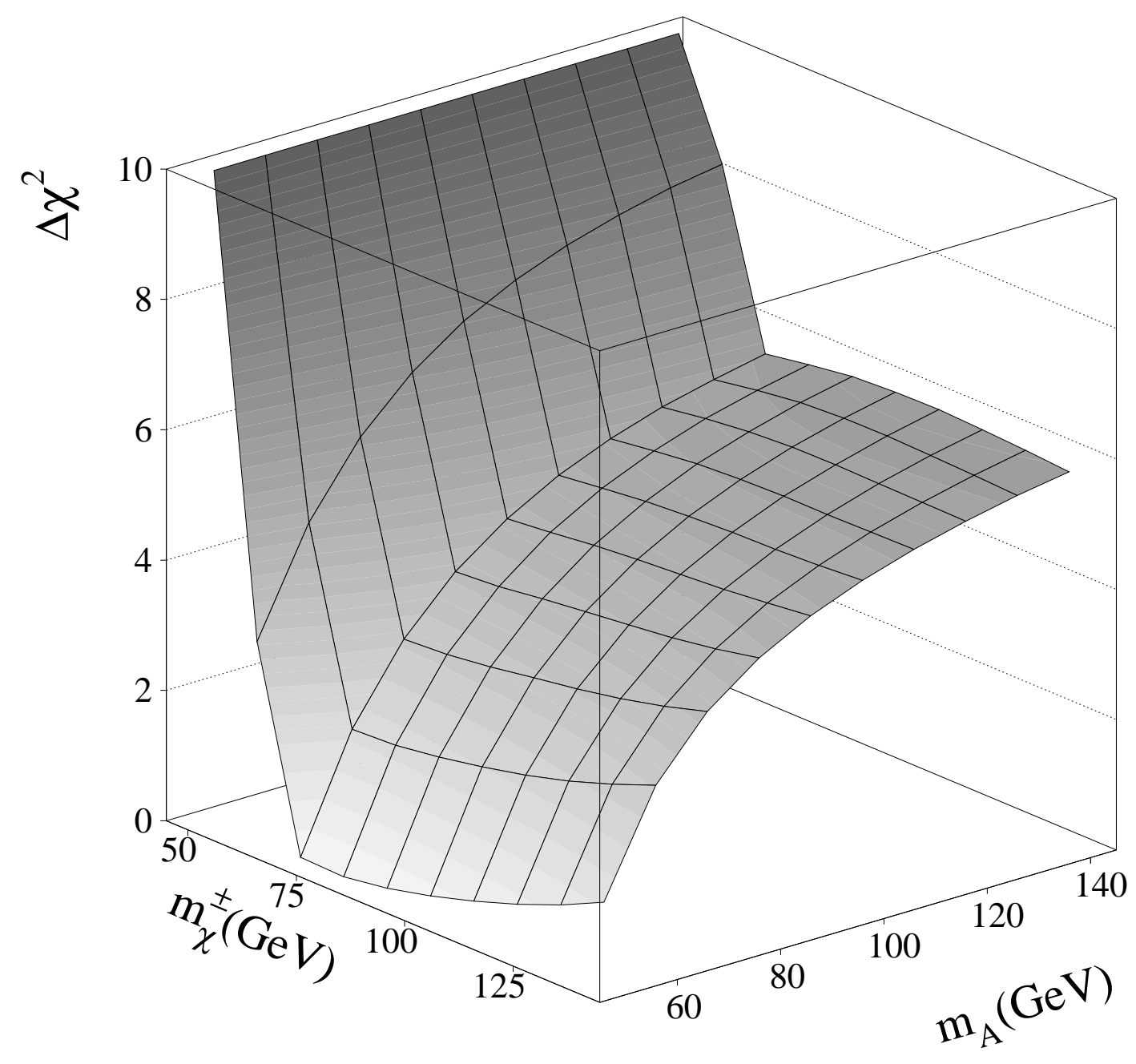

Figure 15: The $\Delta \chi^{2}$ in the pseudo scalar Higgs and light chargino plane for $\tan \beta=$ 50. For each given $m_{A}$ and light chargino mass an optimization of $m_{t}, \alpha_{s}, \tilde{m}_{t 2}$ and the stop mixing angle $\phi_{\text {mix }}$ was performed, including the $b \rightarrow s \gamma$ rate and with the irrelevant parameter $M_{2}$ set to $1500 \mathrm{GeV}$. 


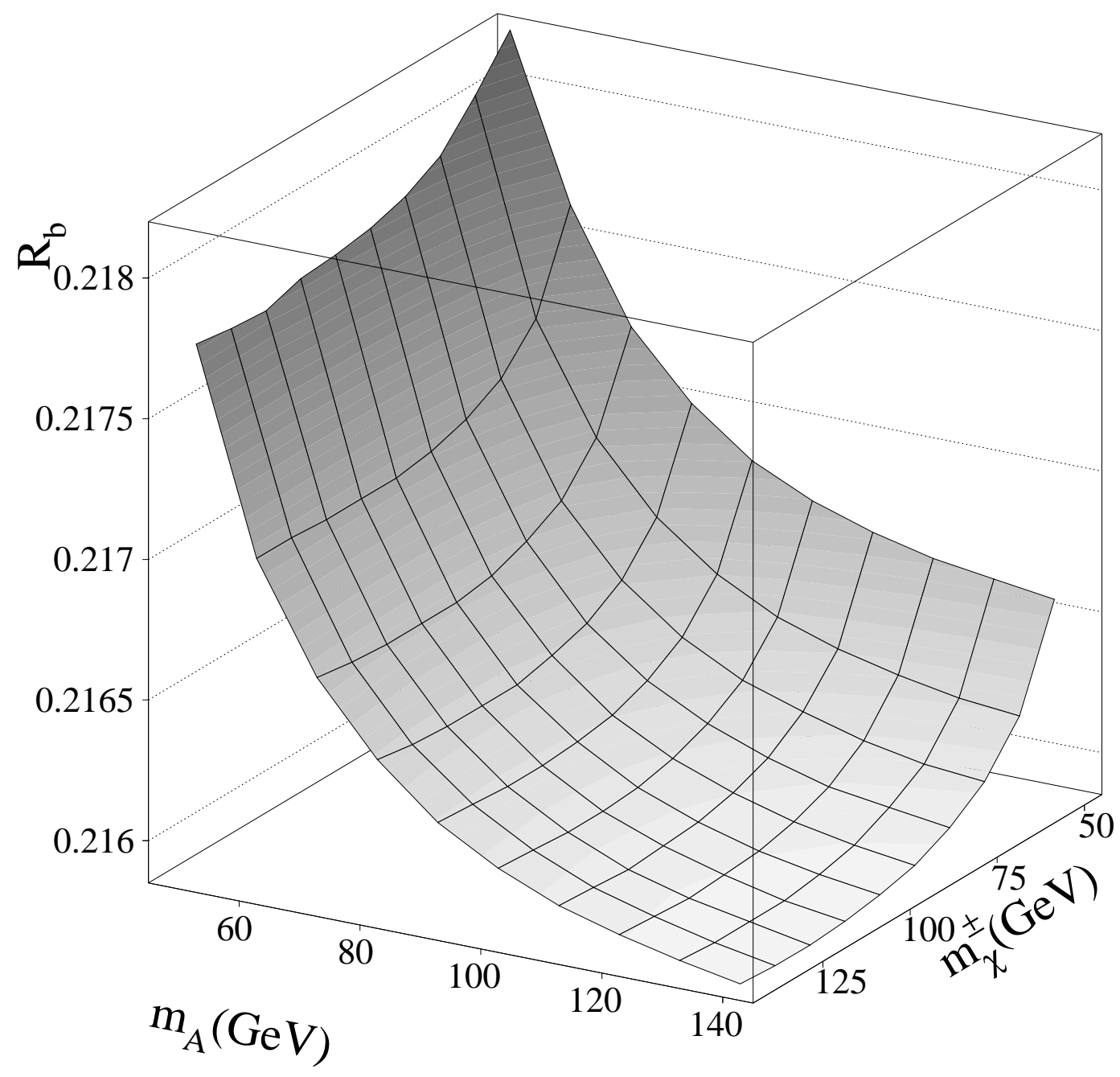

Figure 16: $\quad R_{b}$ in the pseudo scalar Higgs and light chargino plane for $\tan \beta=50$. Optimization as in fig. 15. 


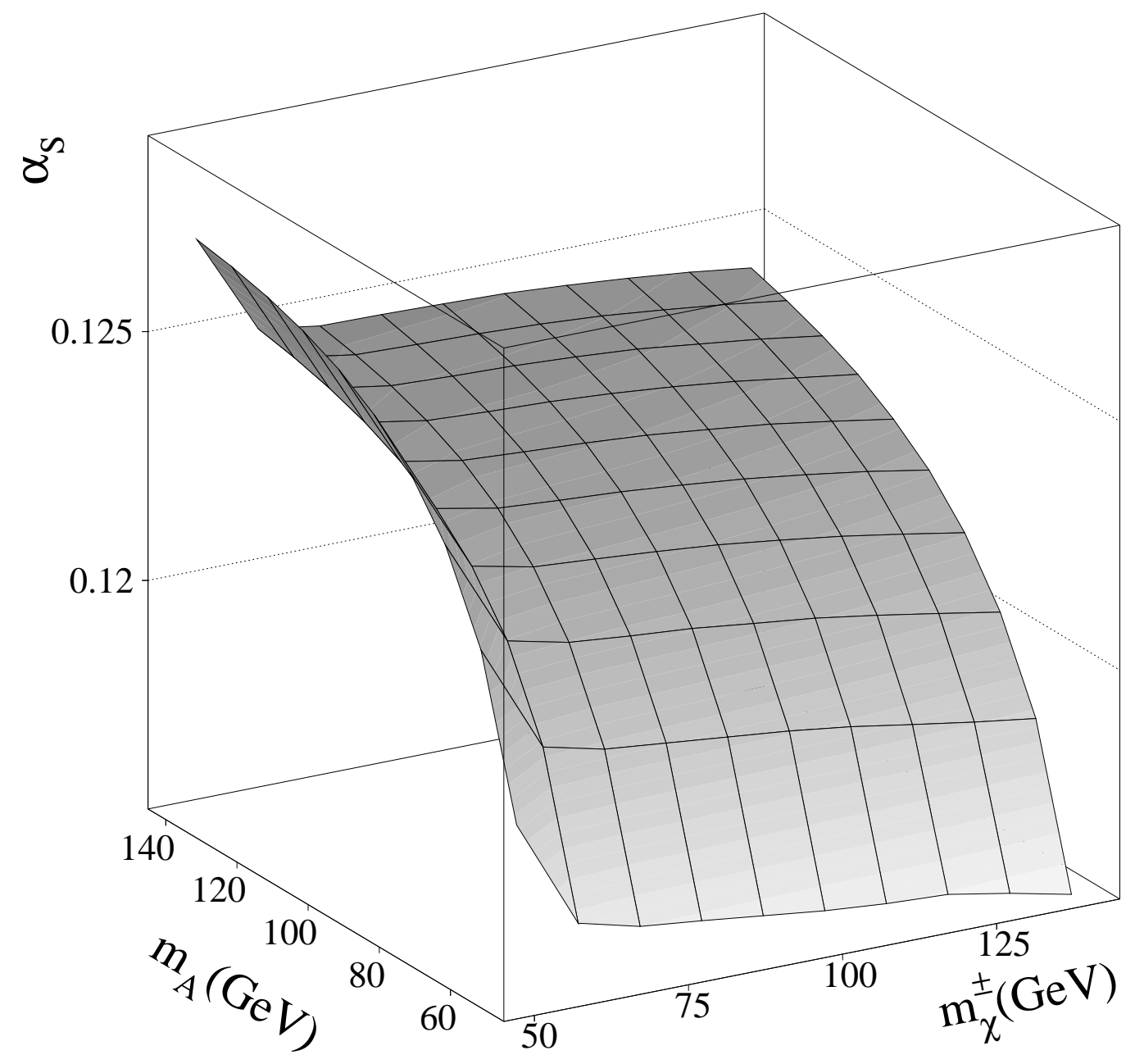

Figure 17: $\alpha_{s}$ in the pseudo scalar Higgs and light chargino plane for $\tan \beta=50$. Optimization as in fig. 15. 


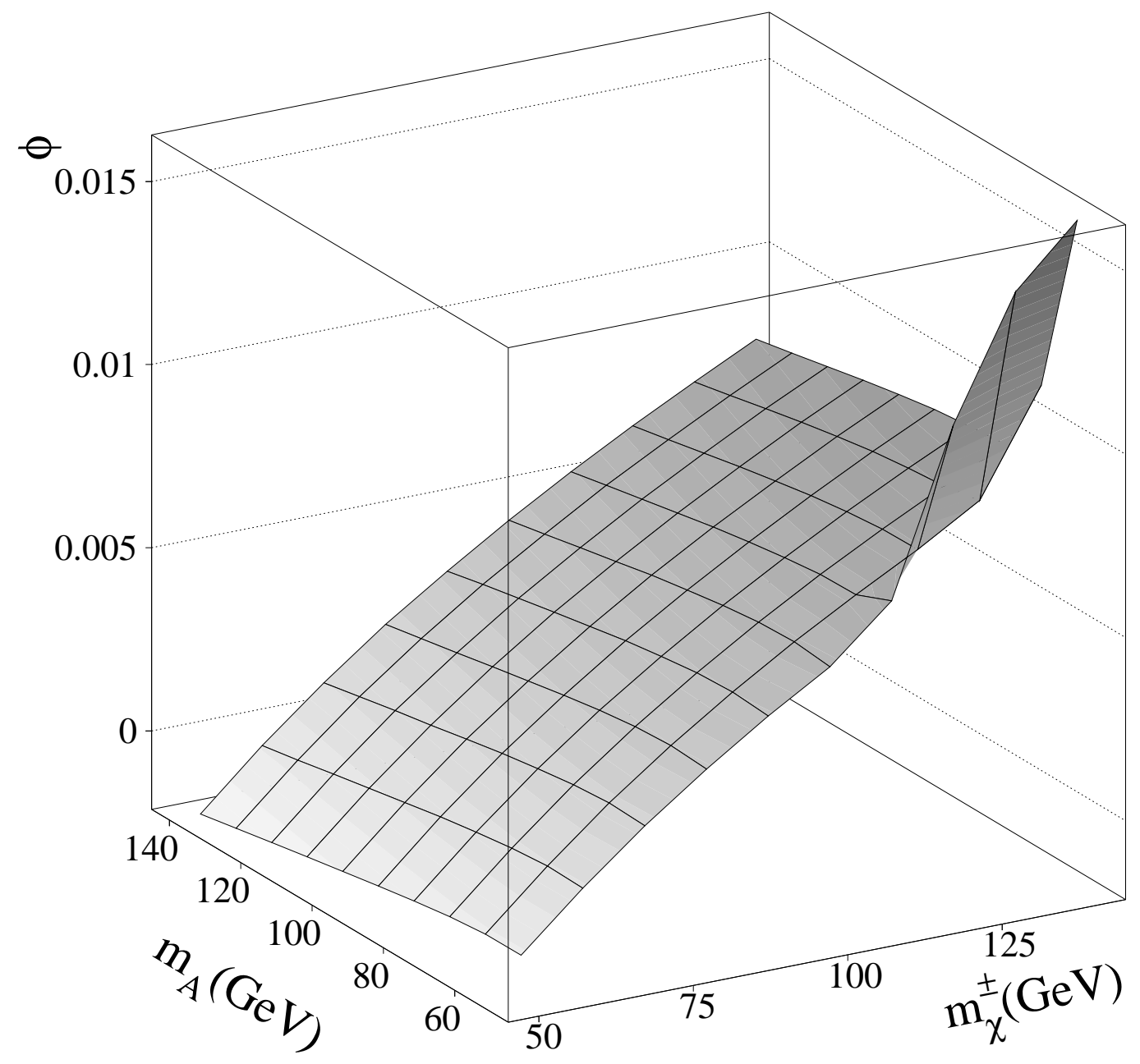

Figure 18: Stop mixing angle $\phi_{m i x}$ in the pseudo scalar Higgs and light chargino plane for $\tan \beta=50$. Optimization as in fig. 15 . 


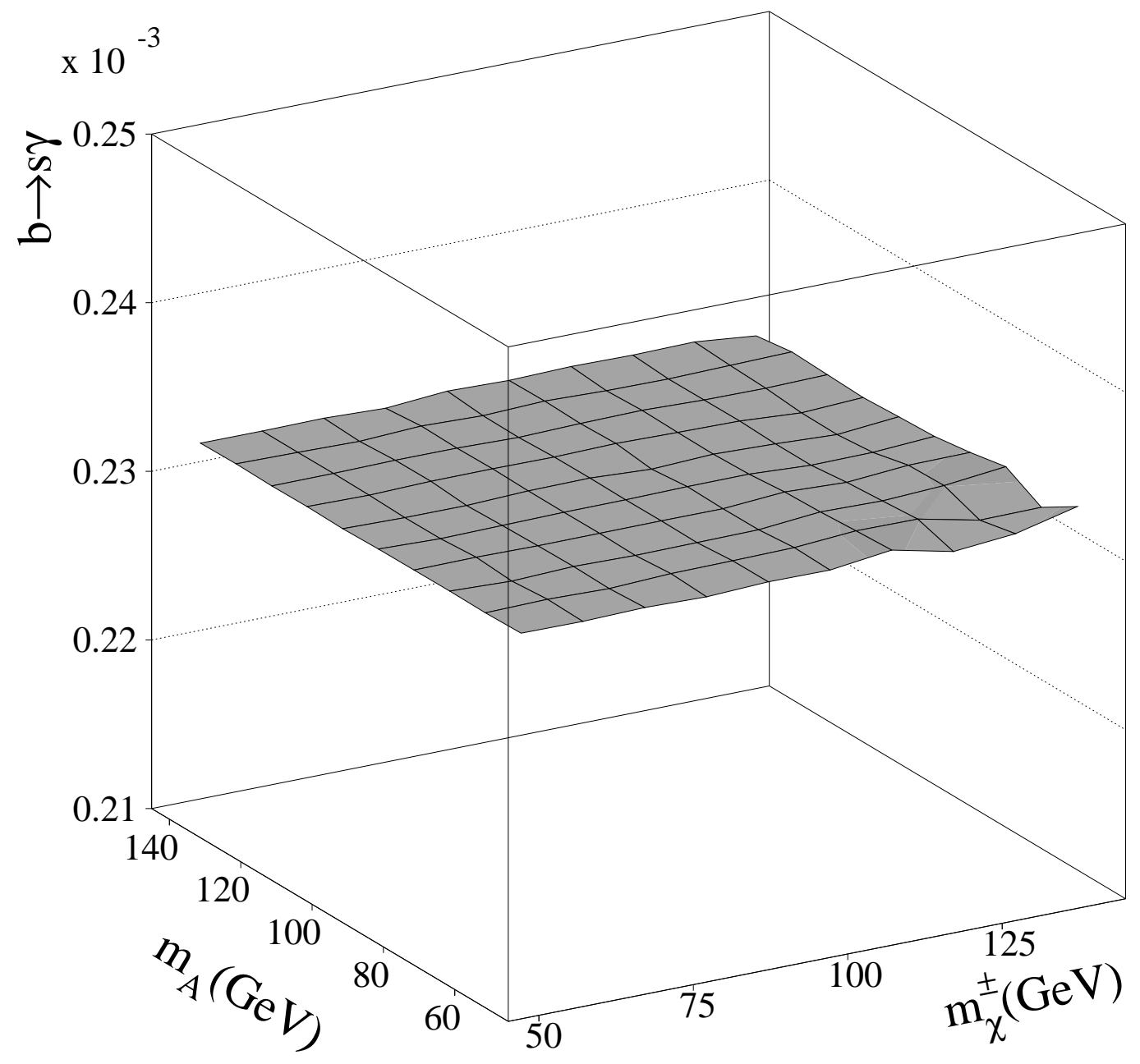

Figure 19: $b \rightarrow s \gamma$ in the pseudo scalar Higgs and light chargino plane for $\tan \beta=50$. The prediction is close to the CLEO measurement of $2.32 \pm 0.67 \times 10^{-4}$ within the whole parameter space. Optimization as in fig. 15. 


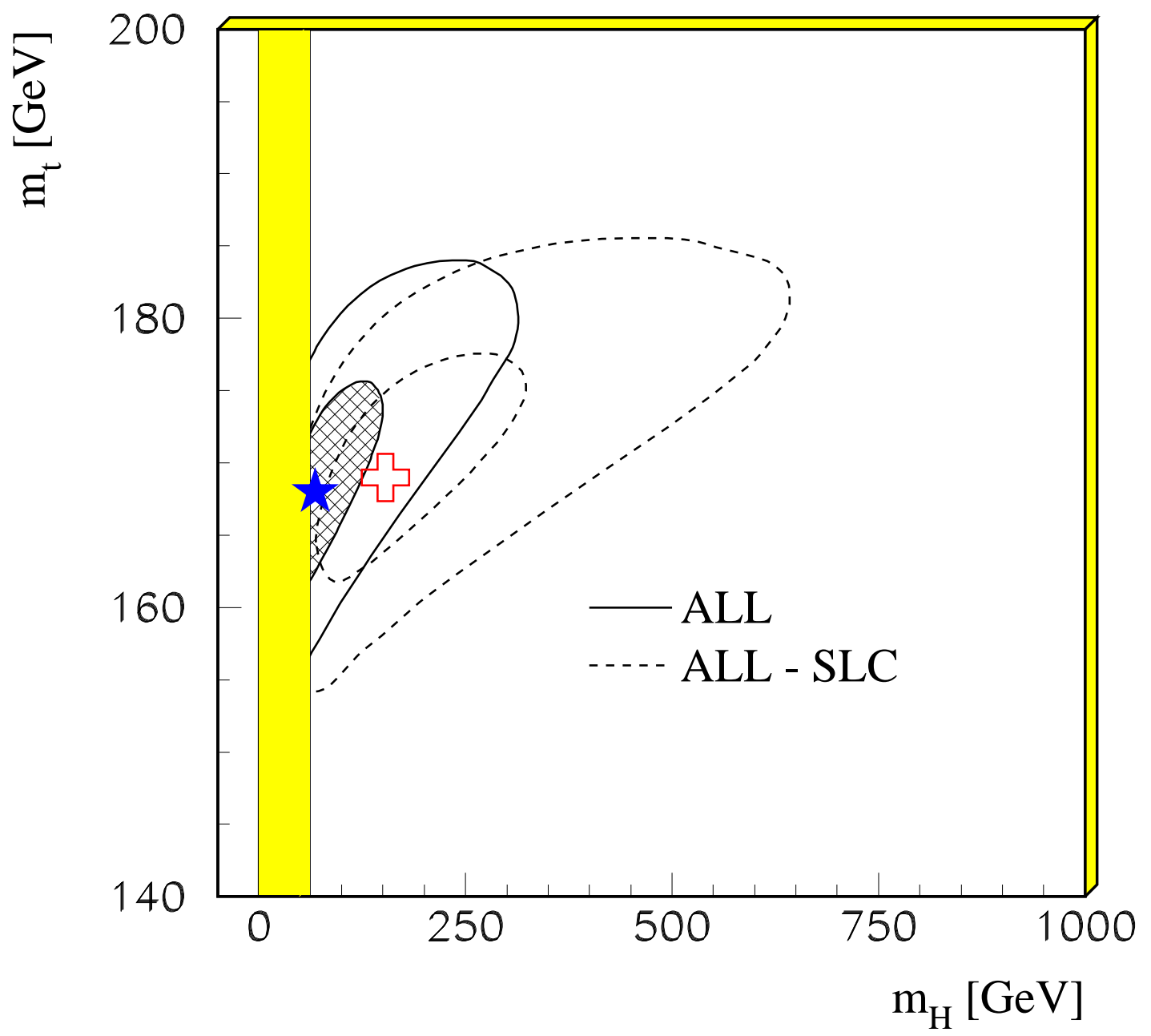

Figure 20: $\Delta \chi^{2}=1$ and $\Delta \chi^{2}=4$ contour lines for all electroweak data including $\sin ^{2} \Theta_{\text {eff }}^{\text {lept }}$ from SLD (continous line) and without it (dashed line). The stars indicate the best fits. 


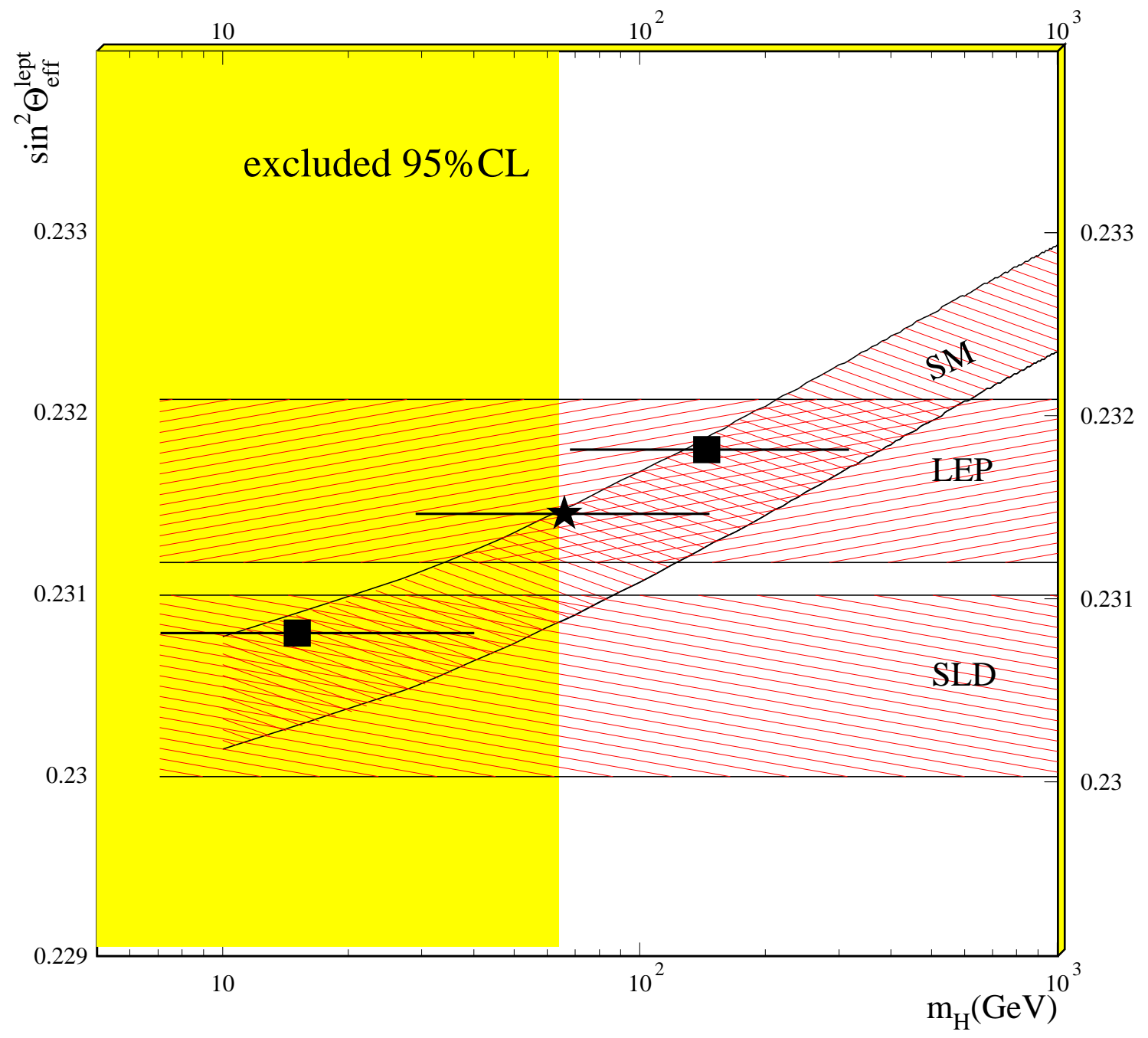

Figure 21: Dependence of the SM $\sin ^{2} \Theta_{\text {eff }}^{\text {lept }}$ on the Higgs mass. The top mass $m_{t}=175 \pm 9 \mathrm{GeV}$ was varied within its error, as shown by the dashed band labelled SM (upper (lower) boundary $m_{t}=166(184) \mathrm{GeV}$ ). The SLD and the LEP measurement of $\sin ^{2} \Theta_{\text {eff }}^{\text {lept }}$ are also shown as horizontal bands. Fits to the electroweak data prefer $m_{t} \approx 170 \mathrm{GeV}$ and light Higgs masses, as indicated by the squares for the separate LEP and SLD measurements, while the star is the result of the combined fit to SLD and LEP data. Clearly, the SLD value yields a Higgs mass less than the recents limits of $63.9 \mathrm{GeV}$ by direct Higgs searches at LEP (shaded area) [19]. 


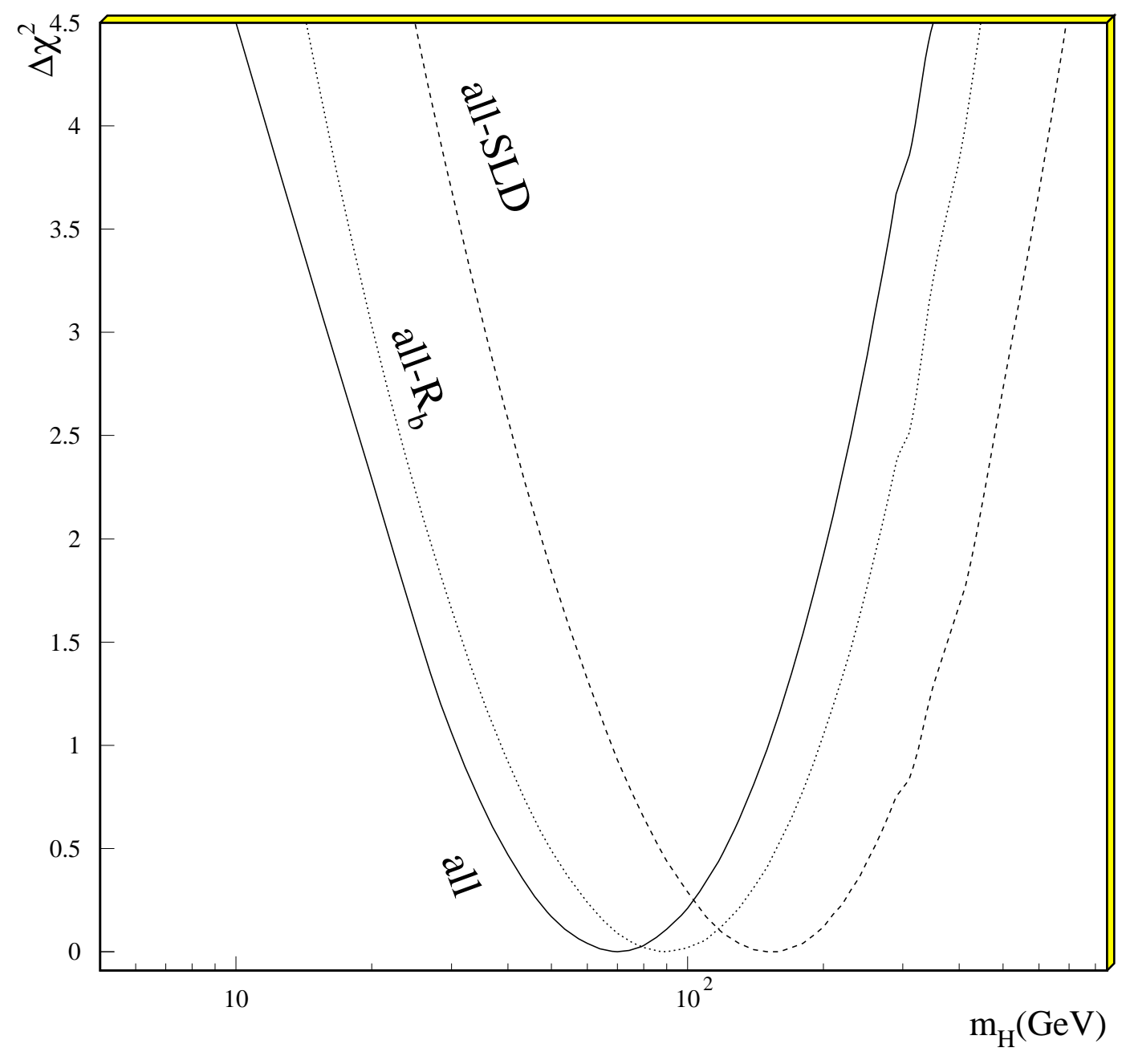

Figure 22: Dependence of the SM $\Delta \chi^{2}$ on the Higgs mass for a free top mass, taking all data (continous line), all data without the SLD measurement of $\sin ^{2} \Theta_{\text {eff }}^{\text {lept }}$ (dashed line) and all data without $R_{b}$ (dotted line). 


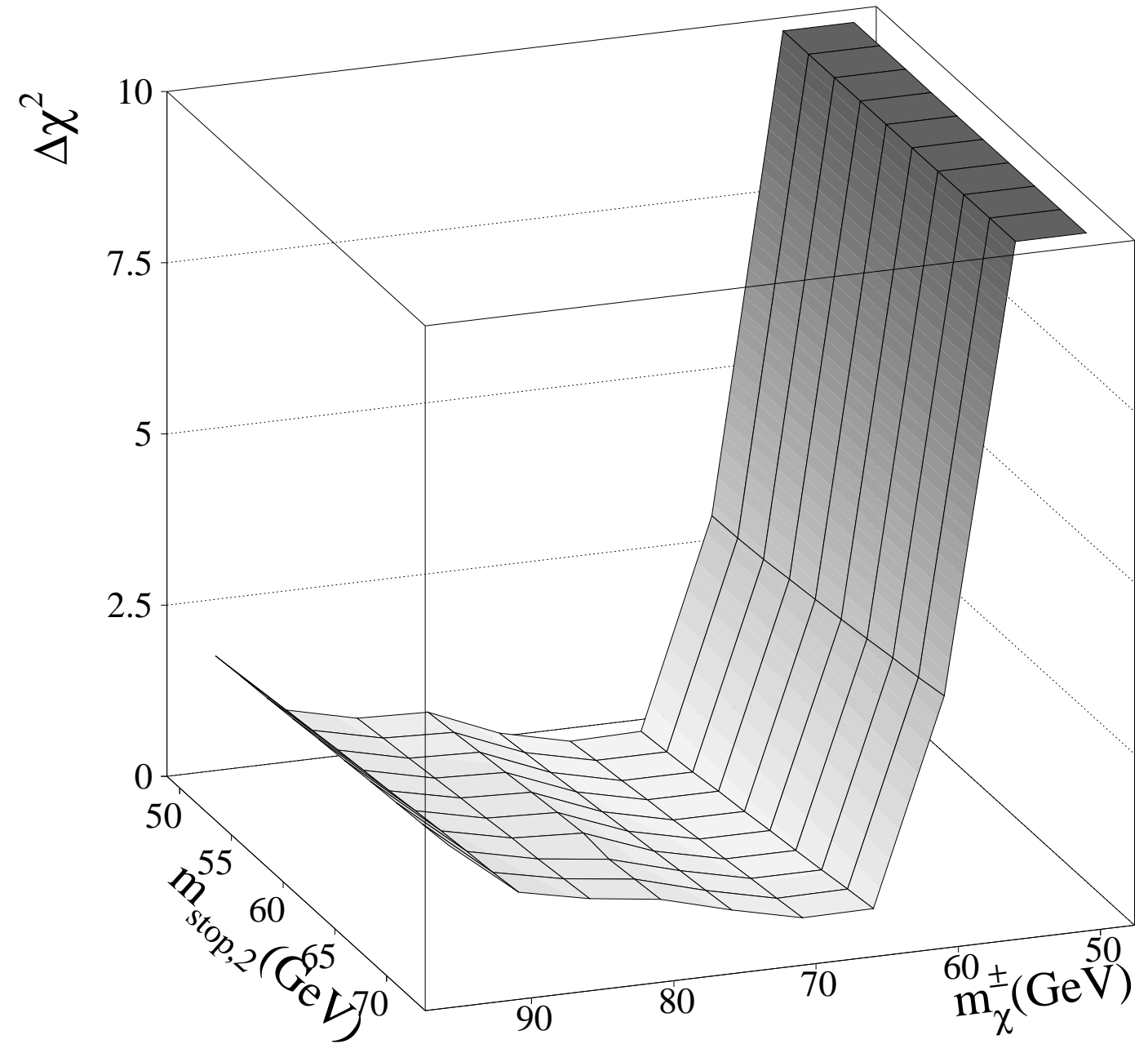

Figure 23: The $\Delta \chi^{2}$ in the region of the best fit in the light stop and light chargino plane for $\tan \beta=1.6$. Here the constraint on $M_{2}$ was dropped. At each point of the grid an optimization of $m_{t}, M_{2}, \alpha_{s}$ and the stop mixing angle $\phi_{m i x}$ was performed with $\mu>-40$, including the ratio $b \rightarrow s \gamma$ and the requirement $\Gamma_{Z \rightarrow \text { neutralinos }}<2 \mathrm{MeV}$. 


\section{Data / MSSM $(\tan \beta=1.001)$ Data $/$ SM}

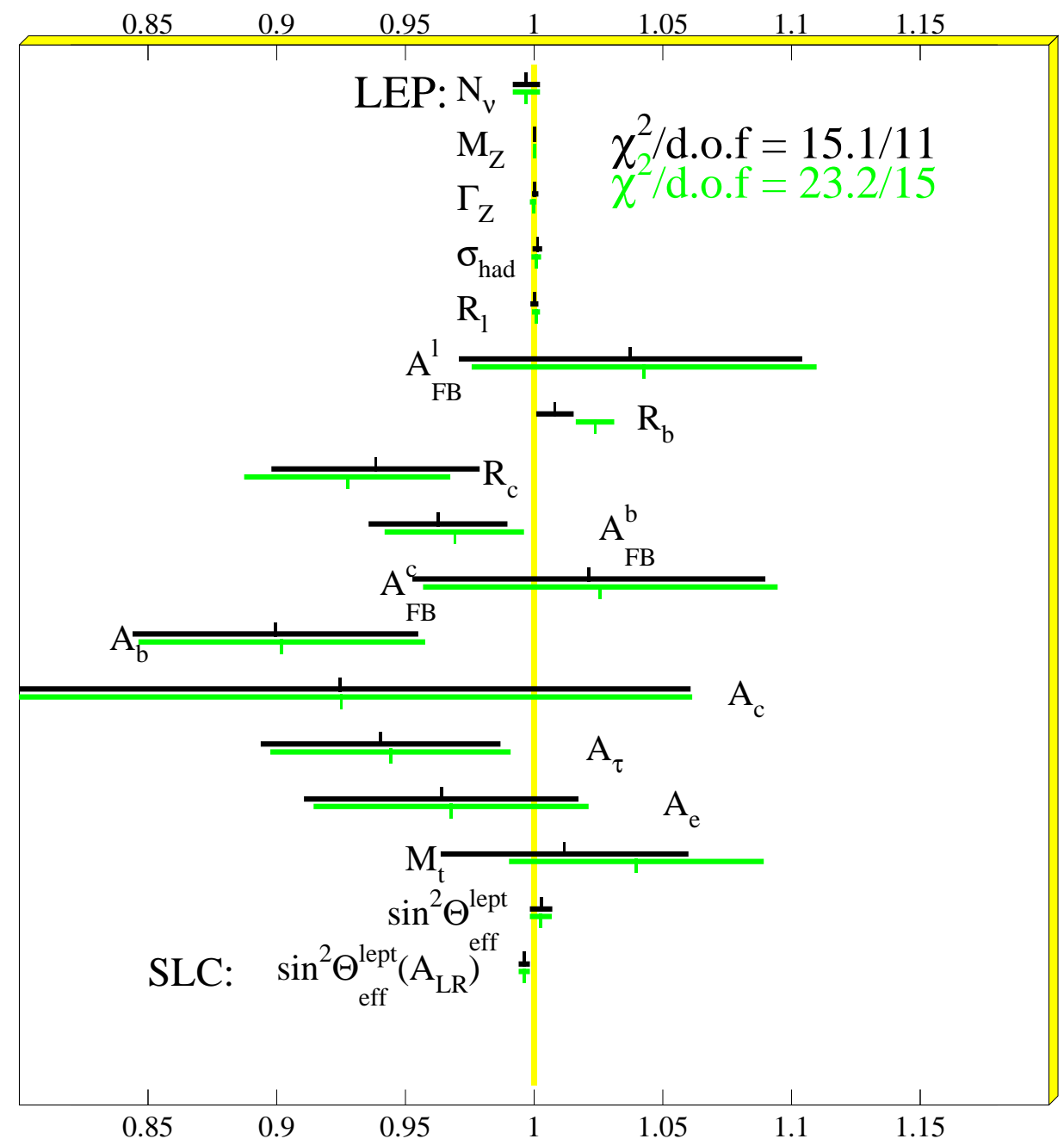

Figure 24: Resulting observables for the fit given in table 3 for $\tan \beta=1.0$. A significant improvement of $R_{b}$ can be observed here. The ratio $b \rightarrow s \gamma$ was not included in this fit here. 


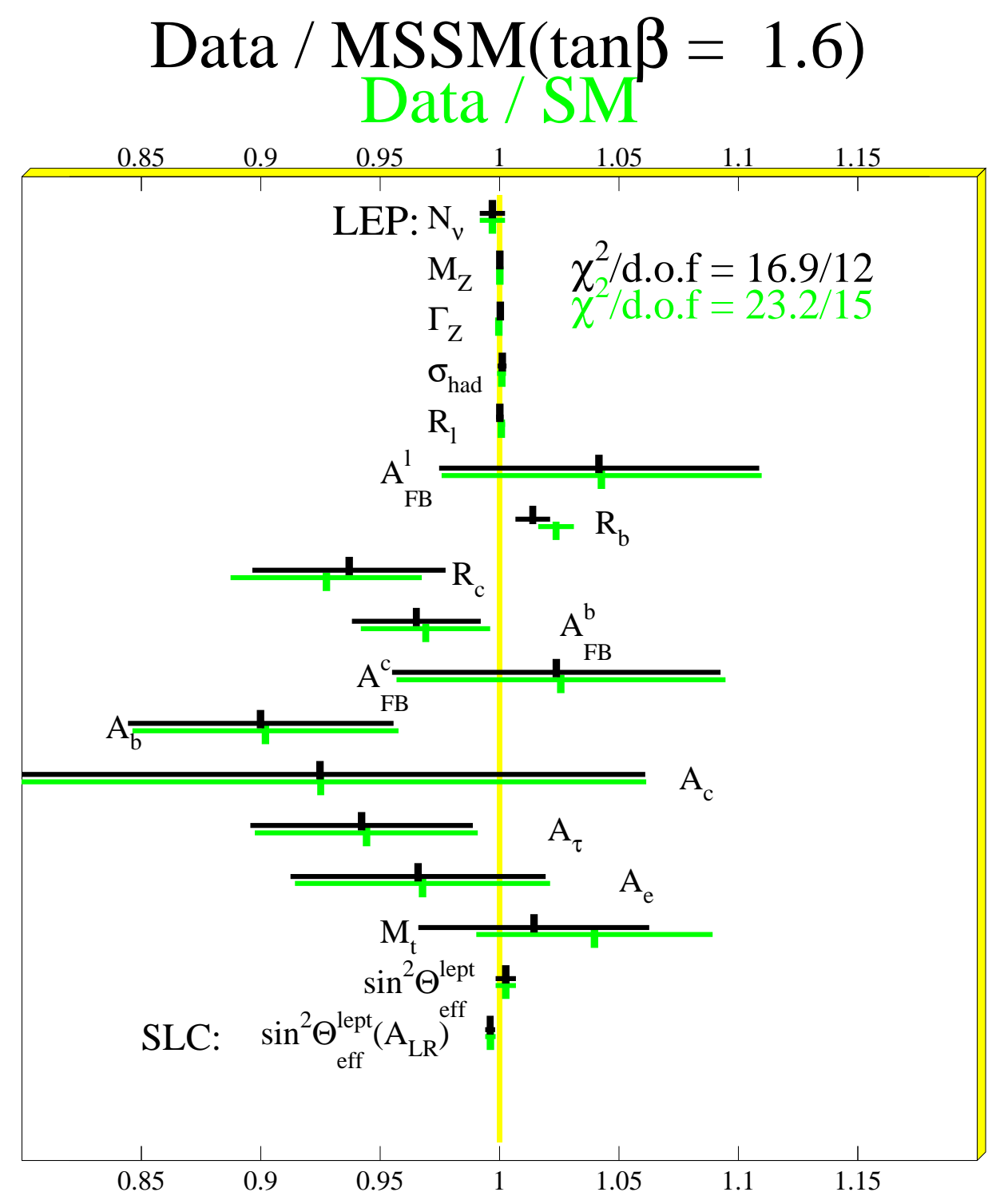

Figure 25: Resulting observables for the fit given in table 3 for $\tan \beta=1.6 . \tilde{m}_{b}$ was fixed to $1000 \mathrm{GeV}, m_{A}$ and the gluino mass were fixed to $1500 \mathrm{GeV}$. Including $b \rightarrow s \gamma$ in the fit it is still possible to improve the prediction of $R_{b}$ with Supersymmetry a bit. 


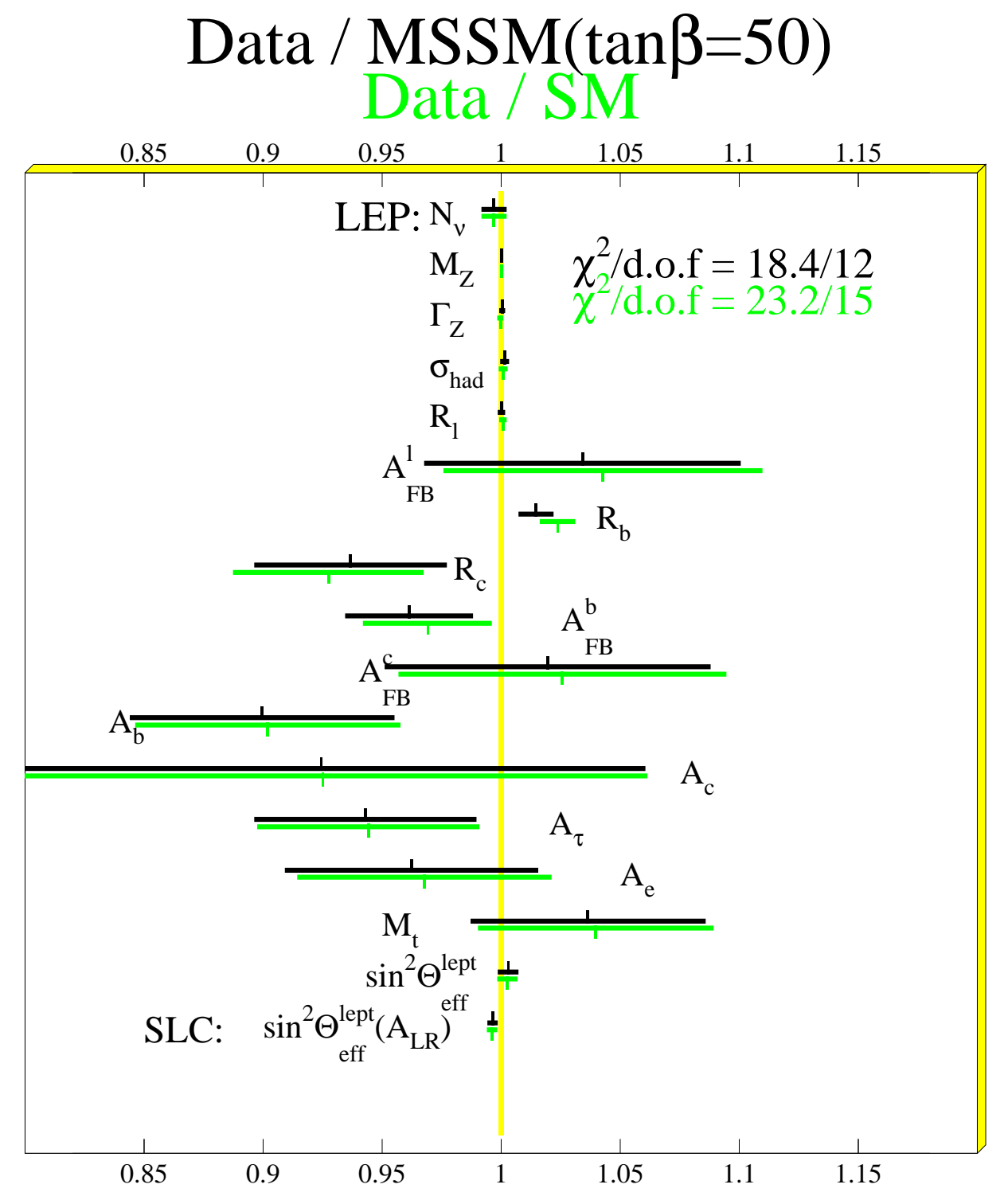

Figure 26: Resulting observables for the fit given in table $\beta$ for $\tan \beta=50 . \tilde{m}_{b}$ was fixed to $1000 \mathrm{GeV}, M_{2}$ and the gluino mass were fixed to $1500 \mathrm{GeV}$. It is possible to improve the prediction of $R_{b}$ with Supersymmetry even for high values of $\tan \beta$, but the result is not as good as for low values. 


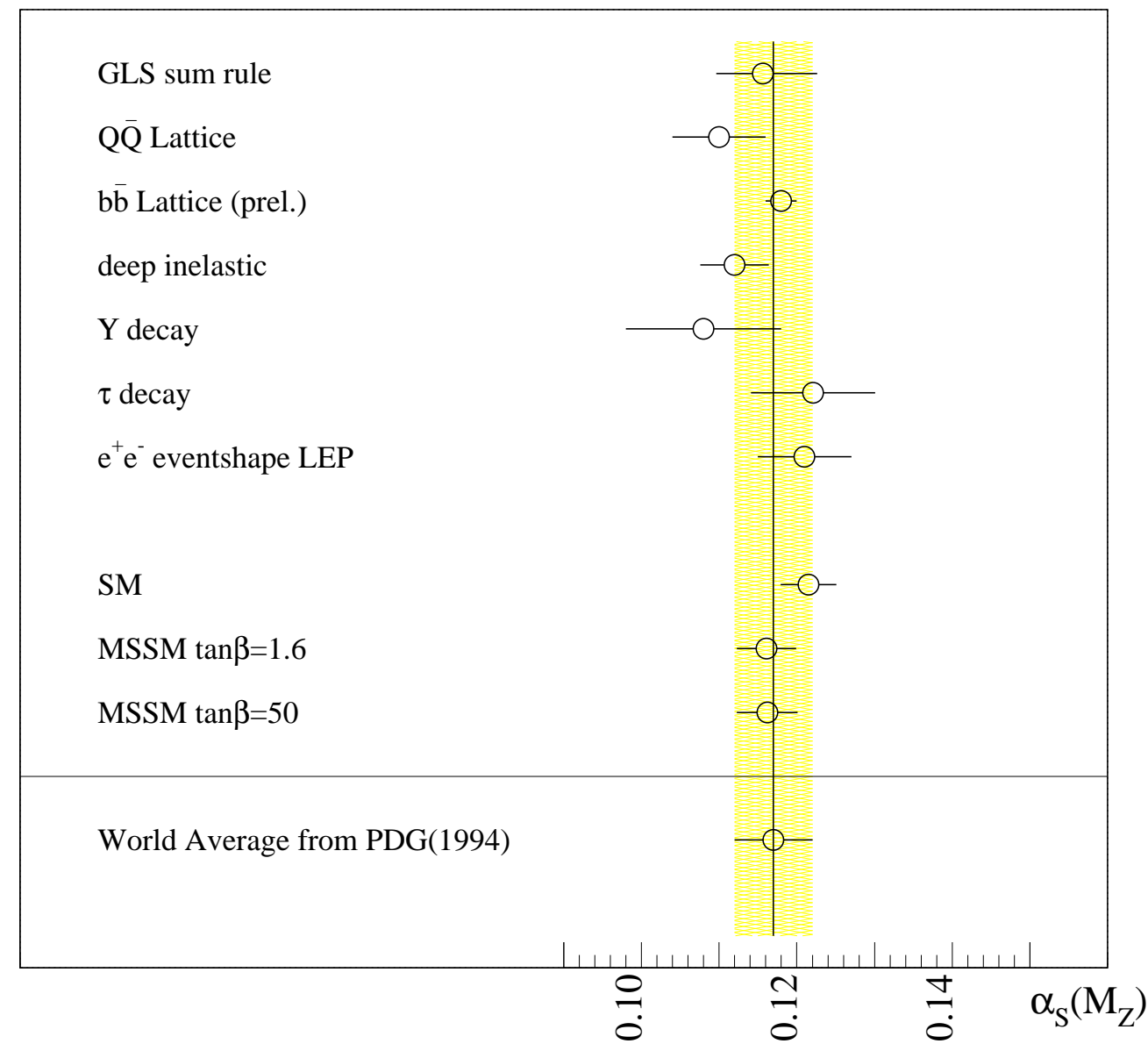

Figure 27: Comparison of different measurements of $\alpha_{s}$ with the fit results. The data has been taken from [20] and [39]. 


\section{References}

1. Proceedings of the Workshop Physics at LEP2, Editors G. Altarelli, T. Sjöstrand, F. Zwirner, Vol.1 and Vol.2, CERN 96-01

2. M. Boulware, D. Finnell, Radiative Corrections to $B R(Z \rightarrow b \bar{b})$ in the Minimal Supersymmetric Standard Model, Phys. Rev. D44(1991) 2054

3. P. H. Chankowski, S. Pokorski, Chargino Mass and $R_{b}$ Anomaly, IFT-96/6, hep-ph 9603310

4. J. Ellis, J. L. Lopez, D. V. Nanopoulos, hep-ph/9512288;

5. D. Garcia, J. Sola, The Quantum Correlation $R_{b}-R_{c}$ in the MSSM: More Hints of Supersymmetry? Mod. Phys. Lett. A9(1994) 211

6. G.L. Kane, R.G. Stuart, J.D. Wells, A Global Fit of LEP/SLC Data with Light Superpartners, Phys. Lett. B354(1995) 350, UM-TH-94-16, hep-ph/9505207

7. J.D. Wells, C. Kolda, G.L. Kane, Implications of $\Gamma(Z \rightarrow b \bar{b})$ for Supersymmetry Searches and Model-Building, Phys. Lett. B338(1993) 219, UM-TH-94-23, hep-ph/9408228

8. D. Garcia, R. Jimenez, J. Sola, Supersymmetric Electroweak Renormalization of the $Z$ Width in the MSSM.Phys. Lett. B347 (1995) 309; Phys. Lett. B347 (1995) 321

9. D. Garcia, J. Sola, Matching the low-Energy and the high-Energy Determinations of $\alpha_{s}(M(Z))$ in the MSSM,Phys. Lett. B357(1995) 349

10. F. James, MINUIT Reference Manual, Version 94.1, Computing and Networks Division CERN Geneva, Switzerland

11. F. Abe et al., CDF Collaboration, Measurements of the W Boson Mass, Phys. Rev. D52 (1995) 4784;

C. K. Jung, DØ Collaboration, W Mass Measurements from D $\varnothing$ and CDF Experiments at the Tevatron, talk given at the 27th ICHEP, Glasgow, Scotland, 20-27 July 1994.

12. F. Abe et al., CDF Collaboration, Phys. Rev. Lett. 74 (1995) 2626, March 1995.

S. Abachi et al., DØ Collaboration, Phys. Rev. Lett. 74 (1995) 2632, March 1995.;

CDF Collaboration, A.Caner, presented at Les Rencontres de Physique de la Vallee d'Aoste, La Thuile, March 1996

D0 Collaboration, M.Narain, presented at Les Rencontres de Physique de La Vallee d'Aoste, La Thuile, March 1996

13. LEP Electroweak Working Group, CERN-preprint LEPEWWG/95-01, March 1995.

14. LEP Electroweak Working Group, CERN-preprint LEPEWWG/96-01, March 1996. 
15. CLEO-Collaboration, R. Ammar et al., Phys. Rev. Lett. 74, (1995) 2885

16. ALEPH Collaboration, Search for supersymmetric particles on $e^{+} e^{-}$collisions at centre-of-mass energies of 130 and $136 \mathrm{GeV}$, CERN-PPE/96-10

17. OPAL Collaboration, Topological Search for the Production of Neutralinos and Scalar Particles, CERN-PPE/96-019;

Search for Chargino and Neutralino Production Using the OPAL Detector at $\sqrt{s}=130-136$ GeV, CERN-PPE/96-020;

DELPHI Collaboration, Search for the Lightest Chargino at $\sqrt{s}=130$ and $136 \mathrm{GeV}$, CERN-PPE/96-75

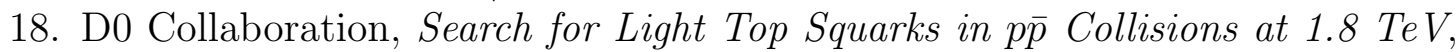
Phys. Rev. Letters 76, 2222 (1996) , FERMILAB-PUB-95/380-E;

DELPHI Collaboration, Search for neutralinos, scalar leptons and scalar quarks in $e^{+} e^{-}$interactions at $\sqrt{s}=130 \mathrm{GeV}$ and $136 \mathrm{GeV}$, in preparation

19. ALEPH Collaboration, Mass Limit for the Standard Model Higgs Boson with the full LEP I ALEPH Data Sample, CERN PPE/96-079

20. Particles and Fields, Phys. Rev. D50 (1994), 1173-1826, Number 3;

R.M. Barnett et al., Phys. Rev. D54 (1996) 1.

21. A. Sirlin, Phys. Rev. D 22 (1980) 971.

W. J. Marciano and A. Sirlin, Phys. Rev. D 22 (1980) 2695.

22. D. Garcia and J. Solà, Mod. Phys. Lett. A 9 (1994) 211.

P.H. Chankowski, A. Dabelstein, W. Hollik, W. Mösle, S. Pokorski and J. Rosiek, Nucl. Phys. B 417 (1994) 101.

23. For a recent review see: Precision Calculations for the $Z$ Resonance, Yellow report CERN 95-03, eds. D. Bardin, W. Hollik and G. Passarino, and references therein.

24. L. Avdeev, J. Fleischer, S. Mikhailov and O. V. Tarasov, Phys. Lett. B 336 (1994) 560.

J. Fleischer, O. V. Tarasov and F. Jegerlehner, Phys. Lett. B 319 (1993) 249;

R. Barbieri, M. Beccaria, P. Ciafaloni, G. Curci. A. Vicere, Phys. Lett. B288 (1992) 95; Nucl. Phys. B409 (1993) 105;

K.G. Chetyrkin, J.H. Kuehn, M. Steinhauser, Phys. Lett. B351 (1995) 331;

J. Fleischer, F. Jegerlehner, P. Raczka, O.V. Tarasov, Phys. Lett. $\mathbf{B 2 9 3}$ (1992) 437;

G. Buchalla, A.J. Buras, Nucl. Phys. B398 (1993) 285

25. A. Dabelstein, W. Hollik, W. Mösle, in preparation.

26. K. G. Chetyrkin, J. H. Kühn and A. Kwiatkowski, Phys. Lett. B282 (1992) 221 ;

K. G. Chetyrkin and A. Kwiatkowski, Phys. Lett. B305 (1993) 285;

K. G. Chetyrkin, A. Kwiatkowski and M. Steinhauser, Mod. Phys. Lett. A 29 (1993) 2785;

A. Kwiatkowski, M. Steinhauser, Phys. Lett. B344 (1995) 359;

K. G. Chetyrkin, J. H. Kühn and A. Kwiatkowski, in: Precision Calculations for the Z Resonance, CERN 95-03, eds. D. Bardin, W. Hollik, G. Passarino 
S. Peris, A. Santamaria, CERN-TH-95-21 (1995).

27. M. Böhm, W. Hollik and H. Spiesberger, Fortschr. Phys. 34 (1986) 687.

W. Hollik, Fortschr. Phys. 38 (1990) 165.

28. A. Denner, R. Guth, W. Hollik, J.H. Kühn, Z. Phys. C51 (1991) 695

29. A. Dabelstein, Z. Phys. C67 (1995) 495; Nucl. Phys. B456 (1995) 25

30. H. P. Nilles, Phys. Rep. 110 (1984) 1.

H. E. Haber and G. Kane, Phys. Rep. 117 (1985) 75.

J. F. Gunion and H. E. Haber, Nucl. Phys. B272 (1986) 1; Nucl. Phys. B402 (1993) 567.

J. F. Gunion, H. E. Haber, G. Kane and S. Dawson: The Higgs Hunter's Guide, Addison-Wesley 1990.

31. J. Ellis, G. Ridolfi and F. Zwirner, Phys. Lett. B257 (1991) 83.

32. R. Barbieri, G. Gamberini, G. Giudice, G.Ridolfi, Signals of Supersymmetry at the $Z_{0}$ Resonance Nucl. Phys. B296 (1988) 75-90.

33. R. Barbieri and G. Giudice, Phys. Lett. B309 (1993) 86;

R. Garisto and J.N. Ng, Phys. Lett. B315 (1993) 372;

S. Bertolini, F. Borzumati, A.Masiero, and G. Ridolfi, Nucl. Phys. B353 (1991) 591 and references therein;

N. Oshimo, Nucl. Phys. B404 (1993) 20;

S. Bertolini, F. Vissani, Z. Phys. C67 (1995) 513, 1995

34. A. J. Buras et al., Nucl. Phys. B424(1994) 374

35. D. Bardin et al., ZFITTER, An Analytical Program for Fermion Pair Production in $e^{+} e^{-}$Annihilation, CERN-TH.6443/92

36. R. Ehret, Die Bestimmung der Kopplungskonstanten $\alpha_{s}$ am LEP-Speicherring und Tests von großen Vereinigungstheorien, Ph.D. Thesis, IEKP-KA/95-13

37. W. de Boer et al., Combined Fit of Low Energy Constraints to Minimal Supersymmetry and Discovery Potential at LEP II, hep-ph/9603350

38. W. de Boer et al., MSSM predictions of the Neutral Higgs Boson Masses And LEP-II Production Cross-Sections, hep-ph/9603346 and references therein

39. B. Grinstein, I.Z. Rothstein Errors in Lattice Extractions of $\alpha_{s}$ due to Use of Unphysical Pion Masses, UCSD-TH-96-09, hep-ph/9605260

40. I. Dunietz, J. Incandela, F.D. Snider, K.Tesima, I. Watanabe, Comments on Recent Measurements of $R_{c}$ and $R_{b}$, FERMILAB-PUB-96/26-T, hepph/9606327

P. Paganini, P. Roudeau, A. Stocchi , An heretic evaluation of the accuracy on $R_{b}$, DELPHI note 96-2 PHYS 586

P. Paganini, P. Roudeau and A. Stocchi, What is really interesting in the measurement of $R_{c}$ ? , DELPHI note 96-66 PHYS 626 


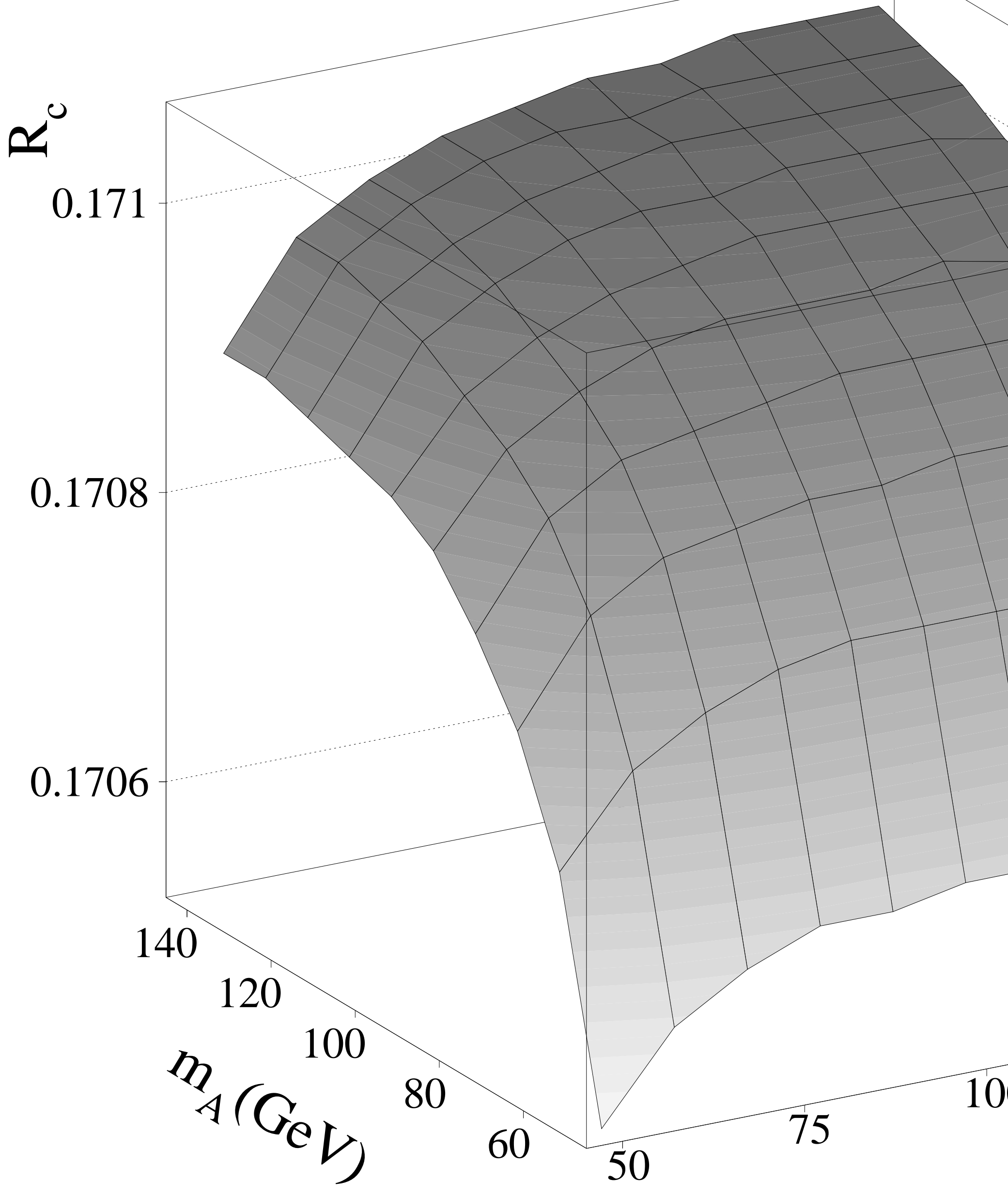




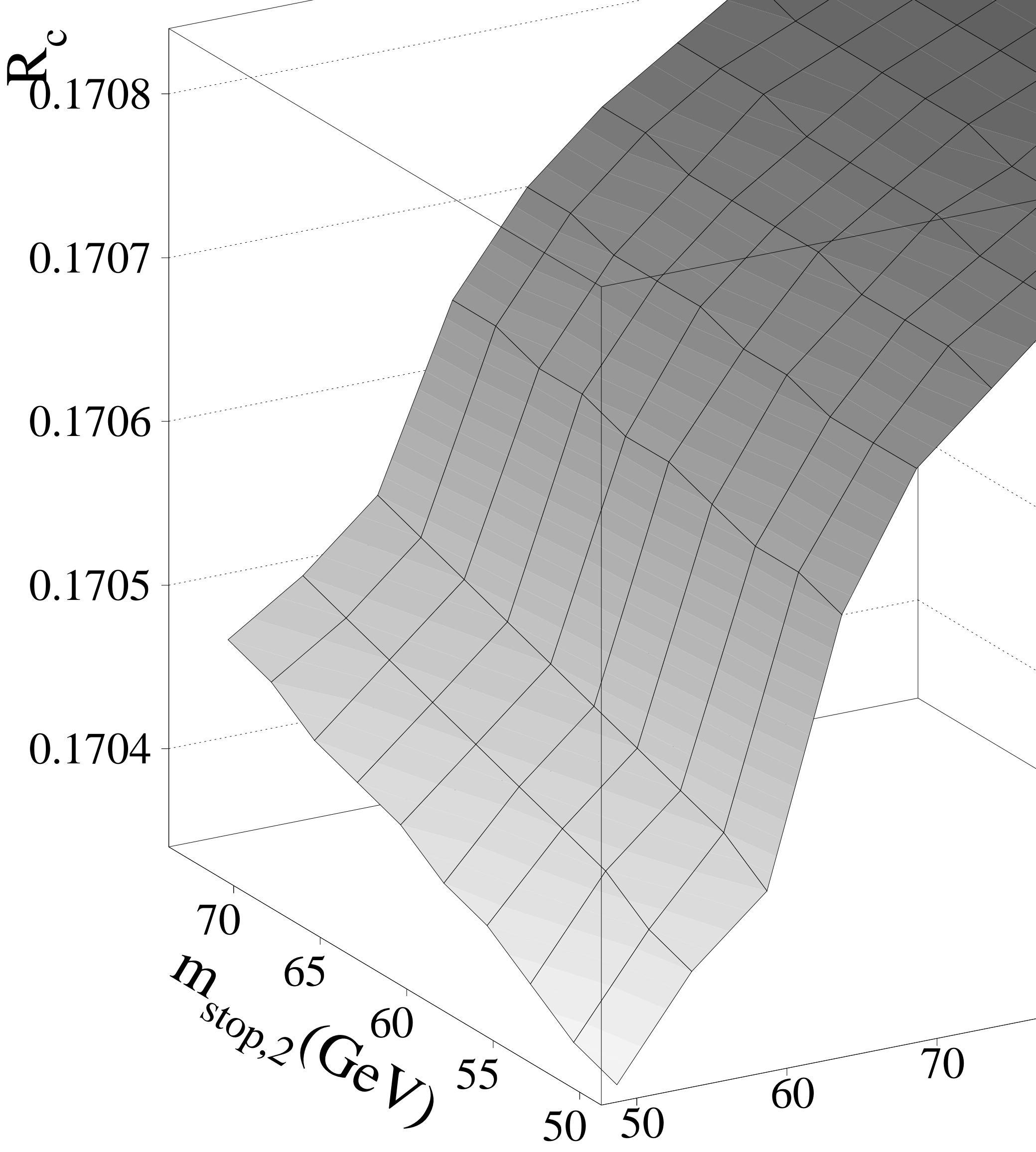

